Portland State University

PDXScholar

Winter 4-11-2016

\title{
A Chemistry Neutral Flow Battery Performance Model Development, Validation, and Application
}

Alasdair James Crawford

Portland State University

Follow this and additional works at: https://pdxscholar.library.pdx.edu/open_access_etds

Part of the Physics Commons

Let us know how access to this document benefits you.

\section{Recommended Citation}

Crawford, Alasdair James, "A Chemistry Neutral Flow Battery Performance Model Development, Validation, and Application" (2016). Dissertations and Theses. Paper 2753.

https://doi.org/10.15760/etd.2754

This Thesis is brought to you for free and open access. It has been accepted for inclusion in Dissertations and Theses by an authorized administrator of PDXScholar. Please contact us if we can make this document more accessible: pdxscholar@pdx.edu. 
A Chemistry Neutral Flow Battery Performance Model

Development, Validation, and Application

\title{
by
}

\author{
Alasdair James Crawford
}

A thesis submitted in partial fulfillment of the requirements for the degree of

\author{
Master of Science \\ in \\ Physics
}

\begin{abstract}
Thesis Committee:
Raj Solanki, Chair

Gary Goncher

Shankar Rananavare

Vilayanur Viswanathan
\end{abstract}

Portland State University

2016 


\begin{abstract}
A physical model for redox flow batteries is developed to estimate performance for any chemistry using parameters such as electrolyte conductivity and kinetic rate constants. The model returns the performance as a function of flow rate, current density, and state of charge. Two different models are developed to estimate the current density distribution throughout the electrode in order to evaluate physical performance of the battery. This is done using electrochemical parameters such as conductivity and kinetic rate constant. The models are analytical in order to produce a computationally cheap algorithm that can be used in optimization routines. This allows for evaluating the economic performance of redox flow batteries, and optimization of cost. The models are validated vs data and found to accurately predict performance in a V-V system for a wide variety of operating conditions.
\end{abstract}


$\begin{array}{ll}\text { Abstract } & \text { i }\end{array}$

List of Figures $\quad$ iv

List of Tables

List of Symbols $\quad$ vii

1 Introduction 1

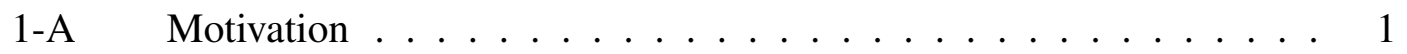

1-B Redox Flow Batteries ................ 2

1-C Cost Modeling .................. 6

$2 \quad$ RFB modeling Basics 11

3 Effective Thickness Model 16

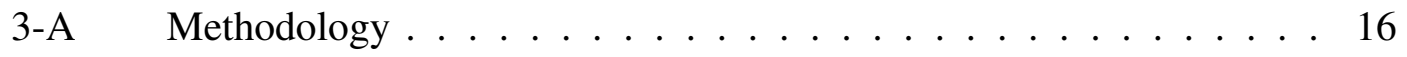

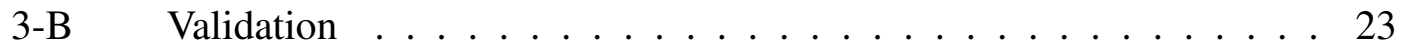

4 Current Distribution Method 28

4-A Methodology ..................... 29

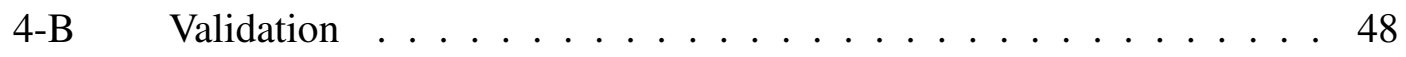

4-C Comparison with Effective Thickness Model .......... 51

$5 \quad$ Application to non-aqueous system $\quad 52$ 


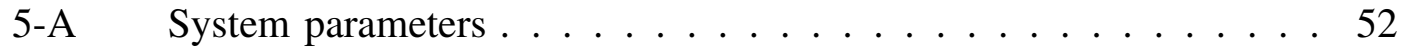

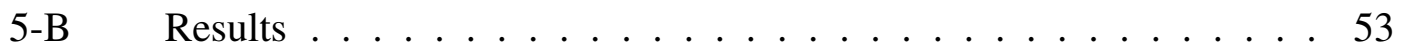

5-C Parametric Analysis ..................... 58

6 Conclusion $\quad 61$

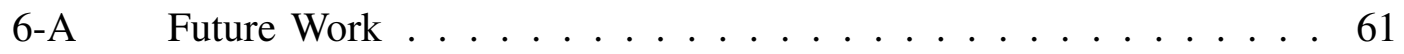

$\begin{array}{ll}\text { References } & 64\end{array}$ 


\section{LIST OF FIGURES}

1.1 Redox Flow Battery Stack Schematic . . . . . . . . . . . . . . 3

1.2 Redox flow battery single cell schematic showing front and side views . . . 4

3.1 The effective thickness of the reaction zone, side view of the cell . . . . . 16

3.2 Effective Thickness Model vs Stack Data for $400 \mathrm{ml} \mathrm{min}^{-1} \ldots$. . . . . . . 24

3.3 Effective Thickness Model vs Stack Data for $800 \mathrm{ml} \mathrm{min}^{-1}$. . . . . . . . . 24

3.4 Effective Thickness Model vs Stack Data for $1200 \mathrm{ml} \mathrm{min}^{-1} \ldots$. . . . . . . 24

3.5 Model vs Stack Voltage for all flow rates and current densities . . . . . . . 25

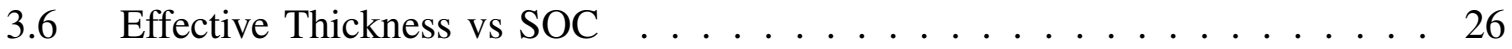

4.1 Current distribution model breaks the cell into sections of thickness h. . . . 29

4.2 The resistor model representing the kinetic and ohmic resistances . . . . . 29

4.3 Current conservation at a single junction . . . . . . . . . . . 30

4.4 A loop of resistors for which Kirchhoff's loop law can be applied to conserve

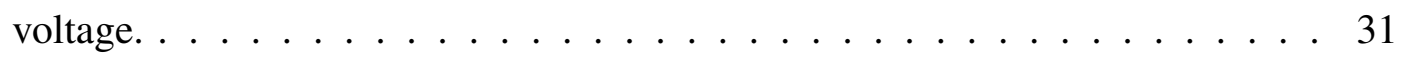

4.5 Error in volumetric current density calculated using first approximation . . . 41

4.6 Error in volumetric current density calculated using integration approxima-

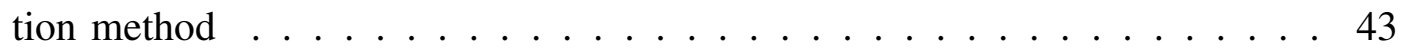

4.7 Curves for $r-1$ vs $b$ giving the same current distribution . . . . . . . . 46

4.8 Volumetric current distributions for various $(r-1) b \ldots \ldots 6$

$4.9 \quad \frac{i_{\max }}{i_{\text {avg }}}-1$ plotted for various values of $\mathrm{r}$ and $\mathrm{b} \ldots \ldots \ldots$

4.10 Current Distribution Model vs Stack Data for $400 \mathrm{ml} \mathrm{min}^{-1} \ldots$. . . . . . . 48

4.11 Current Distribution Model vs Stack Data for $800 \mathrm{ml} \mathrm{min}^{-1} \ldots$. . . . . . 49 
4.12 Current Distribution Model vs Stack Data for $1200 \mathrm{ml} \mathrm{min}^{-1}$. . . . . . . . 49

4.13 Current Distribution Model vs Stack Data for all scenarios . . . . . . . . . . 49

4.14 Current Distribution Model vs Stack Data for Novel Electrode Material . . . 50

4.15 Current Distribution Model vs Stack Data for Fe-V Single Cell . . . . . . . 50

5.1 Costs for various chemistries for 0.1 -h case $\ldots \ldots \ldots 5$

5.2 Costs for various chemistries for 12 -h case ............ 55

5.3 Parametric analysis for present case for $0.1-\mathrm{h} \ldots \ldots \ldots$. . . . . 57

5.4 Parametric analysis for present case for $12-\mathrm{h} \ldots \ldots \ldots$ 


\section{LIST OF TABLES}

1.1 Table of Symbols - RFB System Constants . . . . . . . . . . . . . 6

1.2 Table of Symbols - Operating Parameters . . . . . . . . . . . 7

1.3 Table of Symbols - Design Parameters . . . . . . . . . . . . . . . 7

5.1 Optimized parameters for baseline nonaqeuous system . . . . . . . . . 54

5.2 Optimized parameters for $2 \mathrm{M} 2 \mathrm{e}$ nonaqeuous system . . . . . . . 56

5.3 Optimized electrode thicknesses for parametric analysis . . . . . . . 57 


\section{LIST OF SYMBOLS}

\begin{tabular}{|c|c|}
\hline Symbol & Quantity \\
\hline \hline$a$ & Specific surface area $\left(\mathrm{m}^{-1}\right)$ \\
\hline$E$ & Concentration $\left(\mathrm{mol} \mathrm{m}^{-3}\right)$ \\
\hline$F$ & Potential $(\mathrm{V})$ \\
\hline$I$ & Faraday's constant $\left(\mathrm{C} \mathrm{m}^{-1}\right)$ \\
\hline$I_{K}$ & Total current $(\mathrm{A})$ \\
\hline$i$ & Volumetric current density between electrode and electrolyte $\left(\mathrm{A} \mathrm{m}^{-3}\right)$ \\
\hline$j$ & Applied current density $\left(\mathrm{A} \mathrm{m} \mathrm{m}^{-2}\right)$ \\
\hline$J$ & Electrolyte current density $(\mathrm{A} \mathrm{m}-2)$ \\
\hline$k$ & Kinetic rate constant $\left(\mathrm{m} \mathrm{s}^{-1}\right)$ \\
\hline$k_{m}$ & Mass transport coefficient $\left(\mathrm{m} \mathrm{s}^{-1}\right)$ \\
\hline$n$ & Number of electrons \\
\hline$Q$ & Volumetric flow rate $\left(\mathrm{m}^{3} \mathrm{~s}^{-1}\right)$ \\
\hline$R$ & Ideal gas constant $\left(\mathrm{J} \mathrm{K} \mathrm{mol}^{-1}\right)$ \\
\hline$S O C$ & State of charge \\
\hline$T$ & Absolute temperature $(\mathrm{K})$ \\
\hline$t$ & Electrode thickness $(\mathrm{m})$ \\
\hline$\epsilon$ & Ovorosity \\
\hline$\eta$ & Resistivity $(\Omega \mathrm{m})$ \\
\hline$\rho$ &
\end{tabular}




\section{INTRODUCTION}

\section{A. Motivation}

The amount of energy provided by wind and solar sources have increased rapidly worldwide, producing $17 \mathrm{GW}$ of power in $2000 \mathrm{GW}$ and increasing to $310 \mathrm{GW}$ in 2011 , making up 4\% of all power production [6]. However to make wind and solar economical, batteries to even out short term and long term fluctuations in energy consumption and production.

Wind particularly has a high requirement for energy storage, as it produces the most power at night when loads are low, and less during the day during peak hours. Solar produces the most power during the noon while demand is low, with demand for energy ramping up in the early evening as solar generation decreases. The application of moving energy by several hours is referred to as peak shifting, which requires charge and discharge cycles of anywhere from 1 to 12 hours, or longer [11]. Batteries designed for this application require a high energy to power ratio (E/P) and therefore must focus on reducing the energy component cost of a battery system.

Another application is frequency regulation, that requires charging and discharging with a period of anywhere from a few minutes to an hour. Solar and wind both have short term fluctuations, with the former due to cloud cover and weather, and the latter due to fluctuations in wind speed. This requires a low E/P system, and so batteries designed for this application must focus on reducing the power component cost.

For both of these applications, electrochemical methods of storing energy become the most attractive, due to the ability to quickly change power output [11] and the high 
potential for storing energy at a low price.

\section{B. Redox Flow Batteries}

The redox flow battery (RFB) is one promising solution for grid side energy storage. The main difference between RFBs and conventional batteries is that an RFB stores energy in an electrolyte tank separate from the electrochemical cells, as opposed to conventional batteries with all electrolyte stored in the cell reactor volume. During charge or discharge, the electrolyte in the RFB is pumped in a loop through the cells and back into the tank. The design is more akin to a fuel cell in that the electrolyte serves as fuel to be consumed in the reactor. This means that scaling up the energy storage of an RFB system consists of adding more electrolyte (with a larger tank). The rated power can be scaled up by adding more cells or stacks of cells. As the energy and the power components are completely uncoupled, this makes RFB systems very flexible for a range of applications, especially high energy applications. Due to the liquid electrolyte and high surface area of the electrode, the RFB can ramp up power output very quickly to meet demands of the grid.

RFBs also have a long life span with very little degeneration with consecutive cycles. The primary cause of capacity fade is due to crossover of active species across the membrane, which can be reversed by remixing the electrolyte on each side. This allows an RFB to be more cost effective, as its life cycle cost is reduced.

In order to judge the battery's economic performance in terms of cost per unit energy, it is necessary to calculate its physical performance, as it is directly related. Operating the battery at a higher power density requires smaller cells, reducing the power component 
cost, while running it for high energy efficiency requires less electrolyte, which reduces the energy component cost[10]. In general these two concepts are opposed to each other, and so the optimum battery chemistry and operation depends on the energy to power ratio.

A simplified RFB schematic for a stack of cells is shown in Figure1.1, demonstrating how the components of electrolyte, pump, and cell stack work together. In order to model the performance of a system, a single cell is modeled, with all cells within a stack being assumed to perform identically. The schematic for a single cell is given in Figure 1.2. A single redox flow battery cell consists of two half cells separated by a membrane. Each half cell consists of a conductive electrode within an inert flow frame that directs electrolyte flow. The membrane between them allows crossover of inactive species such as $H^{+}$to maintain electrical neutrality in each half cell and allow for the redox reactions to proceed.

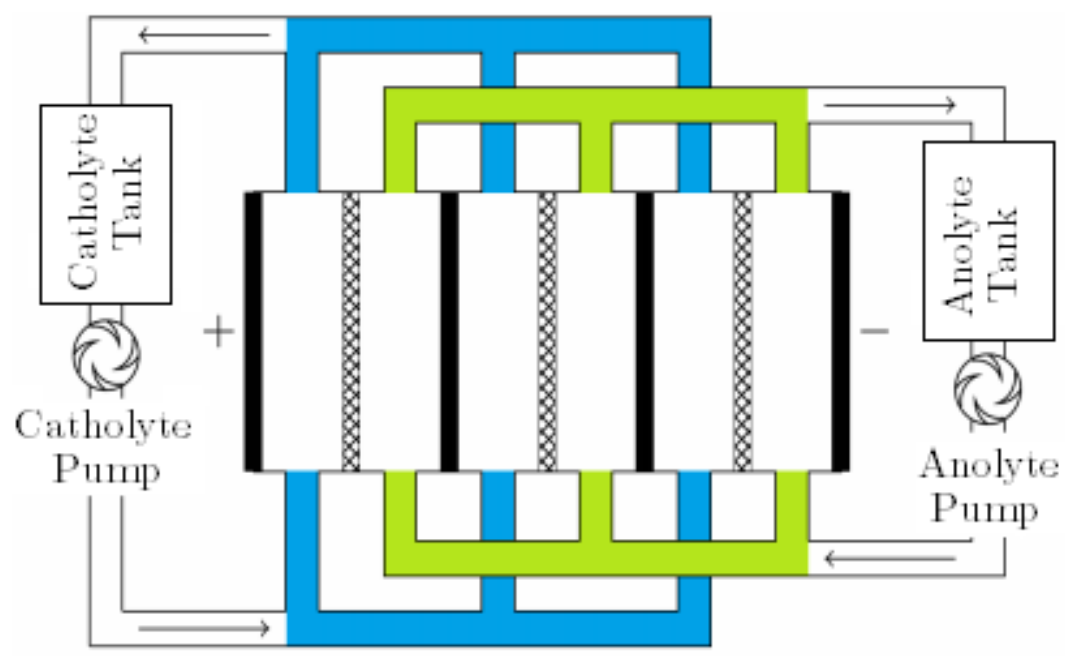

Figure 1.1. Redox Flow Battery Stack Schematic 

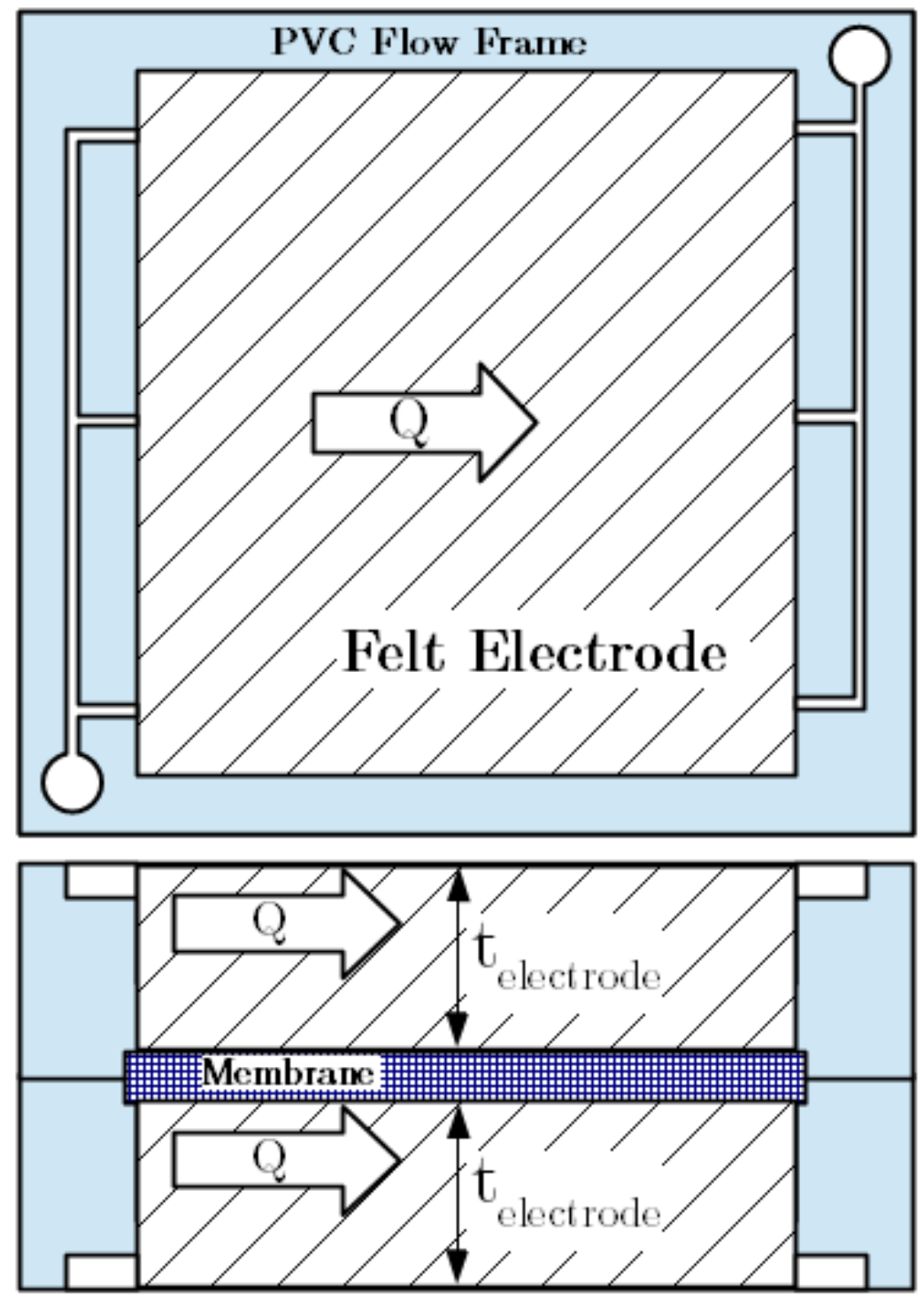

Figure 1.2. Redox flow battery single cell schematic showing front and side views

In this work, a single cell is modeled in order to estimate performance of the entire system. When discussing the single cell, the length dimensions are defined as follows: the thickness refers to the distance between the membrane and the current collector, labeled in Figure 1.2 as $t_{\text {electrode }}$. The height of the electrode refers to the distance along the direction of flow, which is labeled as $Q$ in Figure 1.2. Finally, the width refers to the 
distance perpendicular to the direction of flow. The electrode area refers to the geometrical cross sectional area of the electrode, which is just the height times the width.

There are many possible active species and electrolytes that can be utilized in a flow battery. The type of active species and supporting electrolyte is referred to as battery chemistry. Research at PNNL concentrated on the V-V chemistry with a mixed acid electrolyte[8], consisting of an aqueous solution of hydrochloric acid and sulfuric acid. In the $\mathrm{V}-\mathrm{V}$ system, one side utilizes the reaction $V^{2+} \rightleftharpoons V^{3+}$ while the other side utilizes the reaction $V^{4+} \rightleftharpoons V^{5+}$. The electrode used is a graphite felt, and the membrane is a Nafion proton exchange membrane. The V-V system is of high interest due to its relatively high voltage and energy density, and also can support a high current density. As vanadium is on both sides, the capacity fade from crossover is also minimized. However the cost of vanadium is quite high, and its price can be volatile. The Nafion membrane is also quite expensive at 500-200 $\$ \mathrm{~m}^{-2}$, and is the dominant power component cost.

As a battery cycles, it is convenient to define the state-of-charge (SOC). The SOC corresponds to the fraction of active species that has not yet reacted, hence $100 \%$ SOC corresponds to a completely charged battery with one side being entirely $V^{2+}$ and the other being entirely $V^{5+}$ while $0 \%$ SOC corresponds to one side being entirely $V^{3+}$ and the other side being entirely $V^{4+}$. In practice $0 \%$ and $100 \%$ SOC are never attained, and so the SOC range refers to the difference between the high SOC and the low SOC attained during a cycle. 


\begin{tabular}{|c|c|c|c|}
\hline Symbol & Quantity & V-V Value & Units \\
\hline \hline$E_{0}$ & Standard Cell Potential & 1.3 & $V$ \\
\hline$C$ & Active species total concentration & 2000 & $\mathrm{~mol} \mathrm{~m}^{-3}$ \\
\hline$\epsilon$ & Felt porosity & 0.95 & 1 \\
\hline$k$ & Kinetic Rate Constant & $4-7 \mathrm{e}-6$ & $\mathrm{~m} \mathrm{~s}^{-1}$ \\
\hline$k_{m}$ & Mass Transport Constant & $3 \mathrm{e}-4$ & $\mathrm{~m} \mathrm{~s}^{-1}$ \\
\hline$\rho_{a}, \rho_{c}$ & Anolyte, Catholyte ionic resistivity & $2-4 \mathrm{e}-2$ & $\Omega m$ \\
\hline \multicolumn{2}{r}{ Table 1.1 } \\
\hline
\end{tabular}

TABlE OF Symbols - RFB SyStem CONSTANTS

\section{Cost Modeling}

The PNNL flow battery team developed a cost model based on physical modeling to provide a cost for an RFB system for a given power and energy requirement [10]. This model is coded in $\mathrm{C}++$, is open source and available on GitHub for the battery community to use and investigate[3].

The goal of the cost model is to analyze a given chemistry's battery performance across the SOC range during discharge to calculate the energy and power costs, and then optimize this to determine the lowest cost. As this analysis can be repeated for various energy to power ratios, chemistries can be fairly compared and their optimum applications identified.

Battery performance, and hence cost is very sensitive to operating parameters. For example changing the current density can drastically affect the energy efficiency and thus the energy cost, and also directly affects the power density and thus the power $\operatorname{cost}[10]$.

Therefore to properly compare and analyze battery chemistry, a cost function was developed to produce cost as a function of operating parameters for a given desired energy and power. The primary operating parameters used as input are current density 


\begin{tabular}{|c|c|c|}
\hline Symbol & Quantity & Units \\
\hline \hline$Q$ & Volumetric flow rate per half-cell & $\mathrm{m}^{3} \mathrm{~s}^{-1}$ \\
\hline$j$ & Applied current density & $\mathrm{Am}^{-2}$ \\
\hline$S O C$ & State Of Charge & 1 \\
\hline
\end{tabular}

Table of Symbols - Operating Parameters

\begin{tabular}{|c|c|c|}
\hline Symbol & Quantity & Units \\
\hline \hline$A_{\text {cell }}$ & Cross sectional area of single cell (width times height) & $\mathrm{m}^{2}$ \\
\hline$t_{\text {electrode }}$ & Thickness of half-cell electrode & $\mathrm{m}$ \\
\hline \multicolumn{2}{|c}{ Table 1.3 } \\
TABLE OF SYMBOLS - DeSIGN PARAMETERS
\end{tabular}

at low SOC, flow rate at low SOC, and the SOC range[4]. The cell size is calculated to provide the required power at the low SOC, as performance increases with increased SOC and therefore this cell will be able to provide the required power at all higher SOCs. This cell size is calculated taking into account the power output of the cell, and the losses due to pumping the electrolyte and losses due to parasitic shunt current. This power balance equation is:

$$
P_{\text {req }}=E_{\text {cell }} j A_{\text {cell }}-P_{\text {pump }}-P_{\text {shunt }}
$$

After calculating the size of the cell, this cell area is fixed and the cell's performance across the SOC range is modeled. To do this, the model works backwards starting at the low SOC, where the current density and flow rate are known. Next, the SOC is increased, resulting in increased cell performance and outputting more than the rated power. Hence the flow rate and current density are varied until the power is reduced to the rated power. As increasing the flow rate increases power output due to increased performance, but 
this results in higher losses due to pumping, so the appropriate flow rate is chosen to optimize the net output power. The current density is reduced, maintaining optimum flow rate until the required power output is met. This is done across the entire SOC range. Finally, as voltage is known as a function of SOC, the energy density of electrolyte is calculated from the voltage integrated over the SOC range.

This process allows the cost for the battery system to be determined. The cell area determines the stack component costs, the energy density determines the amount of electrolyte needed, and the flow rate determines the size of pump needed. Hence the system cost is determined as a function of operating parameters.

Next, the cost must be optimized with respect to these operating parameters. In general, increasing the current density increases the power density up to a point, thus reducing the power cost of the battery. However due to the performance reduction, it increases the energy cost. Increasing the SOC range decreases the energy cost as more of the electrolyte is utilized. However it increases the power cost as it is more difficult to attain the required power at the low SOC. Changing the flow rate can allow for increased current and increased SOC range, but can also increase the losses. In general increasing the flow rate is more important for low SOCs, and so higher flow rates are required for energy intensive applications. Hence each of these operating parameters have an optimum value, and this value changes based on whether the application is energy intensive or power intensive. This analysis may be done at multiple energy to power ratios to determine the system cost for various applications, and the best chemistry for each application may be selected. 
The performance model is inside an optimization routine (calculating area and optimizing performance) which itself is inside an optimization routine (optimizing cost), and analysis with in-depth numerical methods such as finite element analysis is not feasible. This is compounded while investigating new chemistries where many different electrochemical parameters may be of interest. An analytical computationally friendly model is necessary for in depth cost modeling and optimization.

It is also desired to produce a model that can analyze novel chemistry. Previous models used empirical fitting factors to estimate performance for $\mathrm{V}-\mathrm{V}$ and Fe-V systems. Models for estimating the ohmic and kinetic losses as well as the change in SOC across the cell only worked when given these empirical fitting factors. The requirement for these fitting factors was hypothesized to be due to the entire electrode not reacting. This means the ohmic drop is changed due to a shorter average distance traveled by the current, and the kinetic losses changed due to a change in the reaction current density. This makes it difficult to use this model when performance data is not available to produce these fitting factors. It also presents difficulties when analyzing the same chemistry with different parameters such as increased surface area or other parametric changes, making it hard to use the model to investigate the effect on cost. Furthermore design parameters such as electrode thickness can be optimized, reducing thickness to deal with low conductivity electrolytes. Finally, this model also is of little when analyzing novel battery chemistries with new active species and electrochemical parameters. Therefore in this work new models are developed to analyze a battery in general from first principles by analyzing the reaction distribution within the cell. 
Two methods are developed and tested for general modeling of RFB performance, improving on previous modeling attempts. 


\section{RFB MODELING BASICS}

When referring to the performance of a flow battery, we are referring to its potential as a function of flow rate, SOC, and current density. From calculating the potential, the power density and energy density can be calculated and used as described in the previous selection. The performance of the flow battery cell can be summarized by dividing it into 4 major components, as in the following equation:

$$
E_{\text {cell }}=E_{0}-E_{\text {nernst }}-\eta_{\text {kinetics }}-\eta_{\text {ohmic }}
$$

The terms are, in order the standard cell voltage $E_{0}$, the Nernst potential $E_{n e r n s t}$, the loss due to charge transfer resistance $\eta_{\text {kinetics }}$ (kinetics), and the loss due to ohmic resistance $\eta_{\text {ohmic }}$. The first term, the Nernst potential is governed by the concentration of the active species at the electrolyte/electrode interface. As these concentrations are mostly governed by the SOC of the system, this part of the performance equation is most sensitive to the current SOC. The Nernst potential is given by:

$$
E_{n e r n s t}=\frac{R T}{n F} \ln \left(\frac{C_{V^{2+}} C_{V^{3+}}}{C_{V^{4+}} C_{V^{5+}}}\right)
$$

Where $\mathrm{R}$ is the ideal gas constant, $\mathrm{T}$ is the absolute temperature, $\mathrm{n}$ is the number of electrons transferred in the half-reaction, and $\mathrm{F}$ is Faraday's constant. $C_{z}$ is the concentration of active species $\mathrm{z}$.

It is convenient to define the term $S O C_{r x n}$ which refers to the SOC at the electrode/electrolyte interface. 


$$
S O C_{r x n}=S O C-\triangle S O C
$$

The SOC refers to the state of charge in the electrolyte tank, which has a volume much larger than the cell reactor volume and is assumed to be constant for a given performance calculation. To calculate the SOC at the reaction interface, there is a drop in SOC due to two factors.

$$
\Delta S O C=\chi_{\text {utilization }}+\chi_{\text {mass transport }}
$$

The first factor is the SOC changing between the inlet and the outlet of the cell as reactants are consumed, which is the utilization. If the whole cell was reacting, this would essentially be the rate at which reactants are consumed divided by the rate at which reactants are pumped into the cell. However this assumption was found to be lacking, drastically under predicting the drop in SOC. Hence in previous models an empirical fitting factor was introduced in to model so that only a fraction of the cell was reacting.

$$
\chi_{\text {utilization }}=\frac{I}{Q C F} \theta
$$

In this utilization equation, $\mathrm{Q}$ is the volumetric flow rate, $\mathrm{C}$ is the total active species concentration, and I is the total current applied to the battery. The numerator is the rate at which the reactants are consumed in terms of Coulombs per second, while the denominator is the rate at which reactants are supplied to the cell. The empirical fitting 
factor is $\theta$. For example, in one analysis the $\mathrm{V}-\mathrm{V}$ model was found to fit best when $\triangle S O C$ was multiplied by 4 , corresponding to $1 / 4$ of the cell reacting.

The second factor is the difference in concentration between the bulk of the electrolyte and the surface of the electrode, given by a mass transport equation [12]. The mass transport equation gives the difference in concentration between the bulk and the surface as a function of current and the thickness of the cell.

$$
\Delta C=\frac{j}{F(a / \epsilon) k_{m}} \frac{1}{t}
$$

Here $\mathrm{j}$ is the applied current density, $\mathrm{t}$ is the thickness of the cell, $\mathrm{a}$ is the specific surface area of the electrode, and $\epsilon$ is its porosity. The mass transport coefficient $k_{m}$ used varies with chemistry, and is based on the diffusivity of the electrolyte and the average distance active species have to travel to the electrode surface from the electrolyte. In the case of graphite carbon felt, this average distance is related to the average distance between carbon fibers.

Note that it is possible for the anode and cathode to have different $S O C_{r x n}$ due to different electrolyte utilization on each side.

In general, increasing flow rate and reducing current density will result in lower $\triangle S O C$ and hence better performance.

This gives the concentration at the electrode-electrolyte interface that govern the Nernst potential and the kinetic losses as:

$$
C_{V^{2+}}=C\left(S O C_{r x n}\right)
$$




$$
\begin{gathered}
C_{V^{3+}}=C\left(1-S O C_{r x n}\right) \\
C_{V^{4+}}=C\left(S O C_{r x n}\right) \\
C_{V^{5+}}=C\left(1-S O C_{r x n}\right)
\end{gathered}
$$

These concentrations are used in the Nernst potential, and are also used in the next term in Equation 2.11, a kinetic term that governs losses due to charge transfer from the reactions.[9]. This is derived from the Butler-Volmer equation, which describes the relationship between the current density and the electrode potential taking into account the anodic and cathodic reaction. Including the losses on the anode and cathode results in the following equation:

$$
\eta_{\text {kinetics }}=\frac{R T}{F} \operatorname{asinh}\left(\frac{j}{2 a F k_{a}} \frac{1}{t_{a} \sqrt{C_{V^{2+}} C_{V^{3+}}}}\right)+\frac{R T}{F} \operatorname{asinh}\left(\frac{j}{2 a F k_{c}} \frac{1}{t_{c} \sqrt{C_{V^{4+}} C_{V^{5+}}}}\right)
$$

In this equation $k$ refers to the kinetic rate constant associated with the anodic and cathodic half-reaction of a specific battery chemistry at a given temperature. The subscripts a and c refer to the quantities on the anodic and cathodic side respectively. Kinetic losses are therefore mostly a function of current density and concentration (and therefore SOC). 
Finally there is an ohmic term that governs losses due to ohmic resistance. This term includes losses from ions crossing the membrane and ions traveling through the electrolyte. Other ohmic losses such as those in the graphite electrode and current collector are considered negligible. The losses due to ohmic resistance follow from ohm's law

$$
\eta_{\text {ohmic }}=j\left(\rho_{m} t_{m}+\rho_{a} t_{a}+\rho_{c} t_{c}\right)
$$

Originally the relevant thicknesses $t_{a}$ for the anode and $t_{c}$ for the cathode were assumed to be the entire cell thickness. However this produced incorrect results unless empirical fitting factors were introduced. This has several effects, for example the ohmic losses become much lower as the entire current density is not traveling through the entire cell thickness. The kinetic losses become higher due to a smaller reaction volume requiring a higher volumetric current density. Finally the change in SOC becomes higher due to the reaction taking place in a smaller volume. Noticing that these parameters are all linked to the same phenomenon, that of the entire cell thickness not reacting uniformly, led to developing a model that took into account reaction distribution to predict performance without fitting factors. 


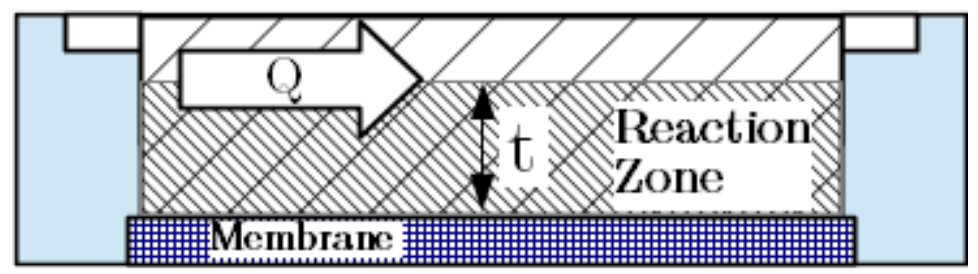

Figure 3.1. The effective thickness of the reaction zone, side view of the cell

\section{EfFective Thickness Model}

\section{A. Methodology}

Building on the previous model, the effective thickness model was developed to calculate the effect of reaction distribution on cell performance, dropping the empirical fitting factors. The core assumption to this model is that within the cell, all the reaction is taking place uniformly inside one reaction zone. Outside of this reaction zone, it is assumed no reaction is taking place. This reaction zone extends from the felt/membrane interface into the felt electrode. Therefore the reaction is assumed to be constant across the cell height and width, and is confined to a rectangular prism within the electrode. This volume can therefore be quantified with just one length factor, referred to as the effective thickness. This is illustrated in Figure 3.1.

This produces a simple expression for the kinetic losses on each side as a function of reaction zone thickness from Equation 2.11. with $\eta_{a, k}$ and $\eta_{c, k}$ referring to the kinetic losses on the anode and the cathode respectively, and $t_{a}$ and $t_{c}$ referring to the effective thickness on the anode and cathode side respectively. 


$$
\begin{aligned}
\eta_{a, k} & =\frac{R T}{F} \operatorname{asinh}\left(\frac{j}{2 a F k_{a}} \frac{1}{t_{a} \sqrt{C_{V^{2+}} C_{V^{3+}}}}\right) \\
\eta_{c, k} & =\frac{R T}{F} \operatorname{asinh}\left(\frac{j}{2 a F k_{c}} \frac{1}{t_{c} \sqrt{C_{V^{4+}} C_{V^{5+}}}}\right)
\end{aligned}
$$

For a given reaction zone thickness, the $\triangle S O C$ is also easily calculated using the utilization term and the mass transport term:

$$
\Delta S O C=\frac{j t_{c e l l}}{Q C F} \frac{1}{t}+\frac{j}{F(a / \epsilon) k_{m}} \frac{1}{t}
$$

This is because for a reaction zone thickness $t$, the fraction of volumetric flow serving this zone is only $t / t_{c e l l}$ where $t_{c e l l}$ is the total cell thickness. The mass transport term is unchanged except for replacing the total cell thickness with the effective thickness.

Using the negative side as an example, this allows the concentrations to be written as:

$$
\begin{gathered}
C_{V^{2+}}=C S O C-\frac{j}{F(a / \epsilon) k_{m}} \frac{1}{t_{a}}-C \frac{j t_{c e l l}}{Q C F} \frac{1}{t_{a}} \\
C_{V^{3+}}=C-C S O C+\frac{j}{F(a / \epsilon) k_{m}} \frac{1}{t_{a}}+C \frac{j t_{c e l l}}{Q C F} \frac{1}{t_{a}}
\end{gathered}
$$

We want to simplify this expression for easy computation of the kinetic losses in terms of reaction zone thickness.

Using the following constants: 


$$
\begin{gathered}
C_{I I}=C S O C \\
C_{I I I}=C(1-S O C) \\
C_{T}=\frac{J}{F(a / \epsilon) k_{m}}+\frac{J t_{c e l l}}{Q F}
\end{gathered}
$$

Plugging this back into the overpotential term, and rearranging:

$$
\eta_{a, k}=\frac{R T}{F} \operatorname{asinh}\left(\frac{1}{\sqrt{\left(\frac{2 a F k_{1}}{j}\right)^{2} C_{I I} C_{I I I} t_{a}^{2}+\left(\frac{2 a F k_{1}}{j}\right)^{2} C_{T}\left(C_{I I}-C_{I I I}\right) t_{a}-\left(\frac{2 a F k_{1}}{j}\right)^{2} C_{T}^{2}}}\right)
$$

The following constants are introduced into Equation 3.9 for ease of computation

$$
\begin{gathered}
A=\frac{R T}{F} \\
B=\left(\frac{2 a F k_{1}}{j}\right)^{2} C_{I I} C_{I I I} \\
D=\left(\frac{2 a F k_{1}}{j}\right)^{2} C_{T}\left(C_{I I}-C_{I I I}\right)
\end{gathered}
$$




$$
G=\left(\frac{2 a F k_{1}}{j}\right)^{2} C_{T}^{2}
$$

This gives the following function for the kinetic overpotential as a function of reaction zone thickness $\mathrm{t}$.

$$
\eta_{a, k}=A \operatorname{asinh}\left(\frac{1}{\sqrt{B t_{a}^{2}+D t_{a}-G}}\right)
$$

Next, the ohmic drop is calculated as a function of reaction zone thickness. Due to the assumption of uniform current distribution, the ions on average travel half of the reaction zone thickness from the electrode to the membrane. This results in the following equation from Ohm's law.

$$
\eta_{a, o}=j \rho_{a} \frac{t_{a}}{2}
$$

Where $\sigma_{a}$ is the ionic resistivity of the electrolyte. Again, for simplicity another constant is introduced to put this in terms of reaction zone thickness:

$$
H=\frac{j \rho_{a}}{2}
$$

This gives the following function for the sum of ohmic and kinetic losses for the negative side as a function of $t$.

$$
f=A \operatorname{asinh}\left(\frac{1}{\sqrt{B t^{2}+D t-G}}\right)+H t
$$


This is our fundamental equation utilized in the effective thickness method. However we do not yet know what the reaction zone thickness is, and have to compute it. The next consideration is that there is a back and forth between the ohmic and kinetics terms. When the reaction zone thickness is high, the ohmic losses are high and the kinetic losses are low, and so the system would force the reaction zone thickness to be lower. If the reaction zone thickness was too high, the kinetic losses would be too high, and so the reaction would spread out more uniformly. Using this physical reasoning, we can assume that just as current follows the path of least resistance, the reaction zone thickness to use should be such that it minimizes the sum of losses $\mathrm{f}$.

From this equation, we notice that there is a minimum possible value of $\mathrm{t}$, which sets the denominator in Equation 3.17 to zero. This represents the case when the reaction thickness becomes so small that the concentration goes to zero due to full utilization, sending the kinetic losses to infinity. This minimum possible effective thickness is given by:

$$
t_{m i n}=\frac{-D+\sqrt{D^{2}+4 B G}}{2 B}=\frac{C_{T}}{C_{I I}}
$$

It is therefore natural to transform this equation using the difference between the minimum value of $\mathrm{t}, t_{\min }$ and the actual value of $\mathrm{t}$. This difference is defined as $\mathrm{x}$.

$$
x=t-t_{\min }
$$

Note that there is also an obvious maximum value of $t$, which is when it is the entire 
thickness of the electrode. Hence we know that the domain of $x$ goes from 0 to $t_{c e l l}-t_{\text {min }}$, which makes it easier to avoid blowing up at the asymptote at $x=0$.

While transforming, we also define the following constant:

$$
\beta=\frac{\sqrt{D^{2}+4 B G}}{B}=C_{T} \frac{\left(C_{I I}+C_{I I I}\right)}{C_{I I} C_{I I I}}
$$

This gives the transformed function we want to minimize:

$$
f=A \operatorname{asinh} \frac{1}{\sqrt{B x(x+\beta)}}+H\left(x+t_{\text {min }}\right)
$$

To minimize this function, we must set its derivative with respect to t to zero. This necessarily corresponds to a minimum, as it has no maximum value, blowing up at the origin. Taking the derivative of Equation 3.21,

$$
\frac{d f}{d x}=A\left(\left(-\frac{1}{2}\right) \frac{1}{\sqrt{B(x+\beta)} x^{3 / 2}}+\left(-\frac{1}{2}\right) \frac{1}{\sqrt{B x}(x+\beta)^{3 / 2}}\right) \frac{1}{\sqrt{1+\frac{1}{B x(x+\beta)}}}+H=0
$$

Rearranging,

$$
\left(\frac{1}{(x+\beta)^{1 / 2} x^{3 / 2}}+\frac{1}{x^{1 / 2}(x+\beta)^{3 / 2}}\right) \sqrt{\frac{B x(x+\beta)}{B x(x+\beta)+1}}=\frac{2 H \sqrt{B}}{A}
$$

On the left hand side, note the term $x^{-3 / 2}$ changes much more rapidly than all the other $\mathrm{x}$ terms. Therefore we can iterate by solving for this $\mathrm{x}$ while assuming the other $\mathrm{x}$ terms are roughly constant, using the previous value of $\mathrm{x}$. That is, 


$$
\left(\frac{1}{\left(x_{i}+\beta\right)^{1 / 2} x_{i+1}^{3 / 2}}+\frac{1}{x_{i}^{1 / 2}\left(x_{i}+\beta\right)^{3 / 2}}\right) \sqrt{\frac{B x_{i}\left(x_{i}+\beta\right)}{B x_{i}\left(x_{i}+\beta\right)+1}}=\frac{2 H \sqrt{B}}{A}
$$

Solving for $x_{i+1}$

$$
x_{i+1}=\left(\frac{2 H \sqrt{B}}{A} \sqrt{\frac{B x_{i}\left(x_{i}+\beta\right)+1}{B x_{i}}}-\frac{1}{x_{i}^{1 / 2}\left(x_{i}+\beta\right)}\right)^{-2 / 3}
$$

Note there as any $\mathrm{x}$ resulting from this is positive, the vertical asymptote at $x=0$ is avoided and there is no possibility of overshoot into negative values of $\mathrm{x}$.

It is necessary to come up with a good initial guess for $\mathrm{x}$. For the first approximation, assume that the argument of asinh is small and $x$ is much smaller than $\beta$, resulting in the following approximation

$$
\operatorname{asinh} \frac{1}{\sqrt{B x(x+\beta)}} \approx \frac{1}{\sqrt{B x(x+\beta)}} \approx \frac{1}{\sqrt{B x \beta}}
$$

This gives the approximate sum of losses function as:

$$
f \approx \frac{A}{\sqrt{B x \beta}}+H\left(x+t_{\min }\right)
$$

Next, take the derivative of Equation 3.27 and set equal to zero to minimize

$$
\frac{d f}{d x} \approx-\frac{1}{2} \frac{A}{\sqrt{B \beta} x^{3 / 2}}+H=0
$$

Therefore the first approximation is: 


$$
x_{0}=\left(\frac{2 H \sqrt{B \beta}}{A}\right)^{-2 / 3}
$$

Therefore the $\mathrm{x}$ that minimizes the sum of losses, can be found by starting with Equation 3.29, then iterating with Equation 3.25. This typically converges to less than $0.1 \%$ error in $4-7$ iterations.

After finding $\mathrm{x}$, the minimum thickness, $t_{\min }$ is added to it to find the effective thickness. This effective thickness is then used in Equation 3.17 to calculate the losses, and Equation 3.3 to find the $\triangle S O C$.

\section{B. Validation}

In order to validate the model, battery performance was calculated using the same operating conditions as a 21 cell stack for which experimental data was available. The 21 cell stack was built and tested internally at PNNL. It was operated at 3 different flow rates, and for each flow rate it was run at 3 different power densities, giving a total of 9 scenarios to test the model.

Overall the model fit the data very well across the various current densities, SOC, and flow rates. The model curves (dashed line) along with the corresponding stack data (solid line) are given for all scenarios in Figures 3.2, 3.3, and 3.4. Note that the y axis goes from $1 \mathrm{~V}$ to $1.5 \mathrm{~V}$, in order to highlight the discrepancy between the model and data. The model performs worse for low flow rates which are not of particular interest for operating the battery, and best at high flow rates. 


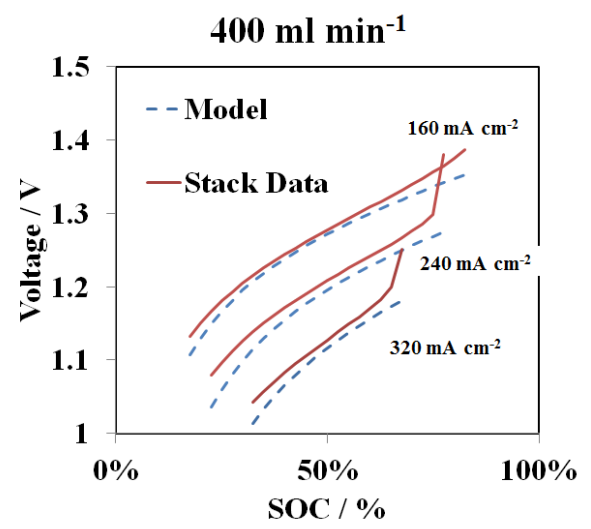

Figure 3.2. Effective Thickness Model vs Stack Data for $400 \mathrm{ml} \mathrm{min}^{-1}$

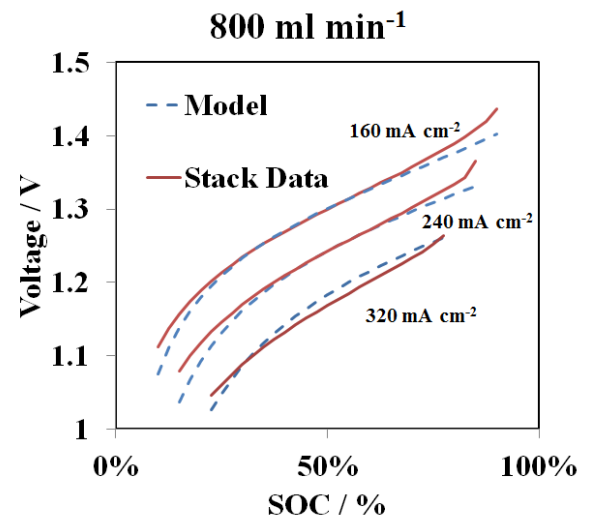

Figure 3.3. Effective Thickness Model vs Stack Data for $800 \mathrm{ml} \mathrm{min}{ }^{-1}$

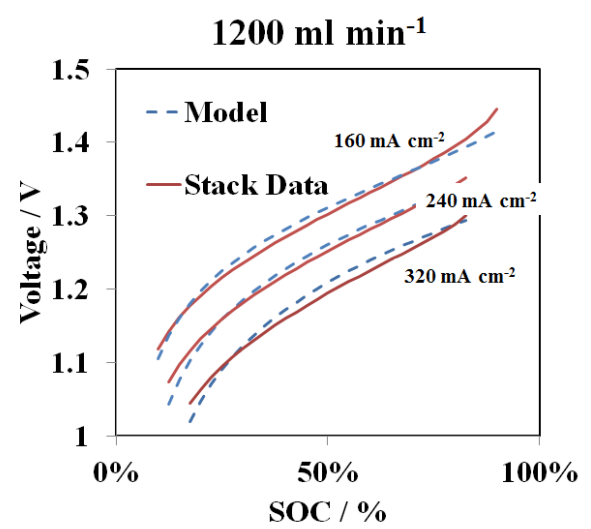

Figure 3.4. Effective Thickness Model vs Stack Data for $1200 \mathrm{ml} \mathrm{min}{ }^{-1}$ 


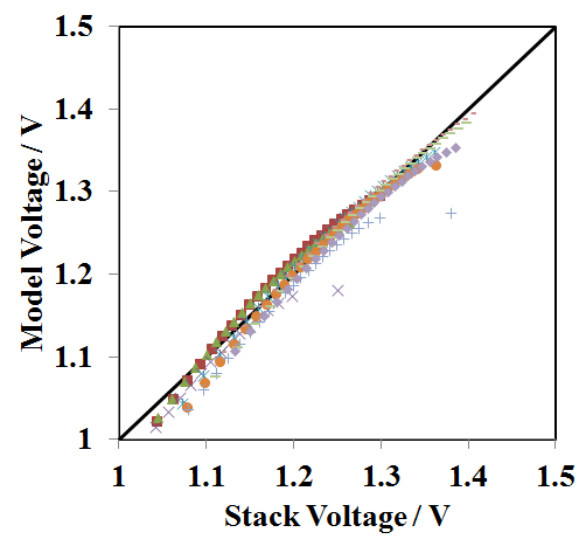

Figure 3.5. Model vs Stack Voltage for all flow rates and current densities

A summary of the model vs the data is given in Figure 3.5, where the model performance is plotted as a function of stack performance at the same SOC. The black line with slope 1 represents where model matching the stack data perfectly, points falling below the line are where the model under predicts and points falling above are where the model over predicts, demonstrating a fairly even distribution. The root mean square error for all scenarios is $16 \mathrm{mV}$.

The two effective thickness curves corresponding to the optimum V-V system for 0.1 $\mathrm{h}$ case and $12 \mathrm{~h}$ case are shown in Figure 3.6. . Note that the effective thickness changes across the SOC range, going from a maximum at low SOC where kinetic losses are highest to a minimum at high SOC. This highlights an advantage of the effective thickness model, that the reaction zone changes with SOC (and current density and flow rate). This contrasts with previous semi-empirical models, where the empirical fitting factors were constants rather than functions of SOC, current density, and flow rate, resulting in a less accurate model.

This model also gives insight into how to choose the thickness of the electrode. If 


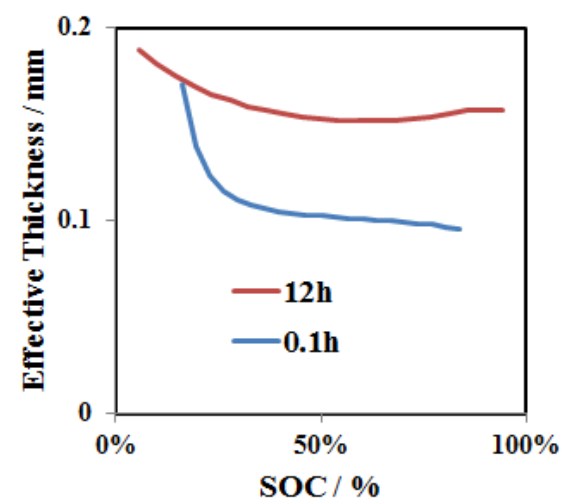

Figure 3.6. Effective Thickness vs SOC

the effective thickness is always thinner than the electrode thickness, this means that a fraction of the electrode thickness is not being utilized, despite energy being expended to pump this electrolyte through the electrode. Energy is wasted on pumping this nonreacting electrolyte, and the drop in concentration from inlet to outlet increases as a smaller ratio of the electrolyte is supporting the reaction. This means we can reduce losses with a thinner electrode. On the other hand, if the effective thickness is equal to the electrode thickness, this could mean that the thickness is too small and kinetic losses are too large, and could be reduced with a thicker electrode. Note also that for a high energy application, the effective thickness is larger due to a smaller current density (reducing the ohmic losses) and a larger SOC range (increasing the kinetic drop at low SOC). Therefore the trend is for a thicker electrode for an application with higher energy requirements, and a thinner electrode for an application with higher power requirements. The thicker electrode allows the SOC range to be pushed, increasing the energy density, while a thinner electrode allows for the power density to be pushed.

The optimized electrode thickness corresponding to lowest cost is based on the highest 
value of the effective thickness at the low SOC. An electrode thicker than this will have electrolyte flowing outside the reaction zone. A thinner cell will result in lower performance due to a higher over potential, as well as an increased pressure drop. By restricting the felt thickness to its value at low SOC, pumping losses can be cut down by $50 \%$ or more, since all the pumped electrolyte is actively participating in the reaction. At low SOC, this allows the flow rate to be increased to mitigate $\Delta \mathrm{SOC}$ related losses. 


\section{Current Distribution Method}

Although the effective thickness model turns out to be an effective method of modeling battery performance, there are some issues. Treating the current density as uniform is a large simplification of the physics, and the approximation may break down under certain conditions. There is also some degree of iteration involved to solve for the effective thickness, which adds to computation time, especially if the solution takes a long time to converge.

Another issue is choosing an electrode thickness. Part of the motivation of building the effective thickness model was to choose an electrode thickness that works best for a given chemistry. The effective thickness model gives some insight, but using it ace value would suggest the best electrode thickness would be the largest effective thickness during cycling. This is due to the effective thickness model's assumption that reactions only take place within the reaction zone, and not outside it - therefore all electrolyte outside the reaction zone costs energy to pump, but is not reacting and is therefore just reducing energy efficiency. However, in reality the reaction should be more spread out, with the effective thickness only an abstraction. Reducing the electrode thickness to the effective thickness would not actually correspond to the optimum electrode thickness. As battery performance can be optimized by choosing the best electrode thickness, we want a model that can properly describe how performance changes with electrode thickness.

Therefore the next logical step in modeling RFBs is to produce a model that properly takes into account the current distribution, thereby modeling the entire electrode thickness by producing a $1 \mathrm{D}$ model. 


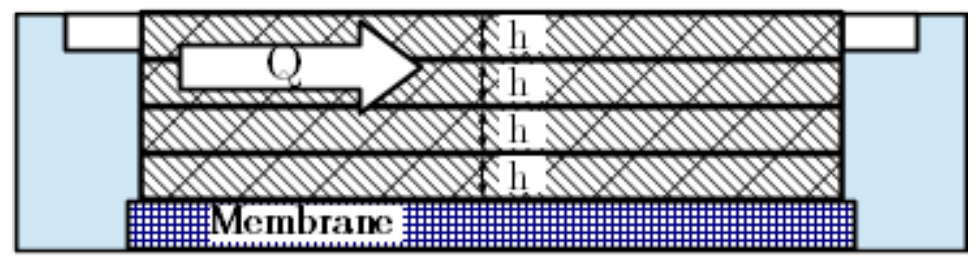

Figure 4.1. Current distribution model breaks the cell into sections of thickness $h$.

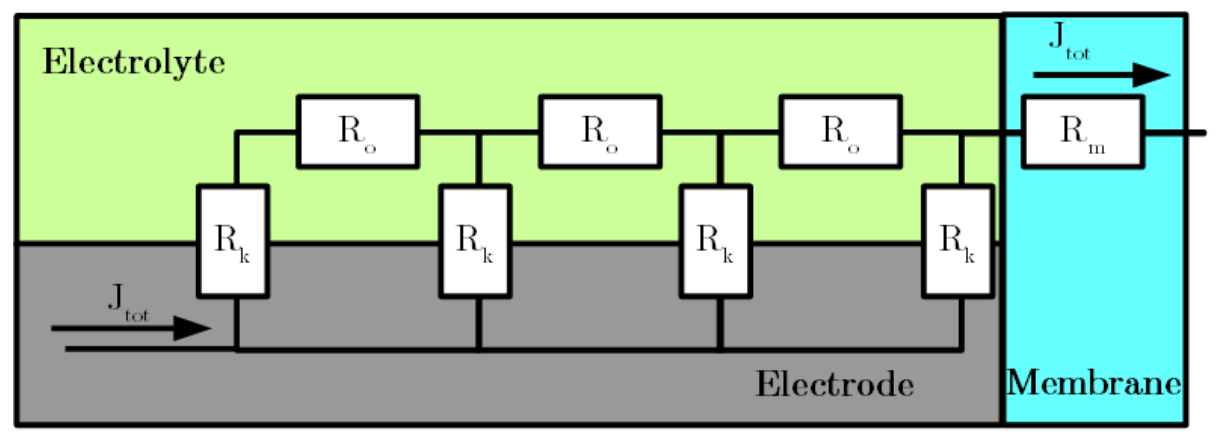

Figure 4.2. The resistor model representing the kinetic and ohmic resistances

\section{A. Methodology}

The current distribution model takes into account variations in current across the electrode. It starts with breaking the electrode down into sections of thickness $\mathrm{h}$, as in Figure 4.1. Each of these sections is treated as having their own associated current densities, one for the ionic current density in the electrolyte traveling towards (or away from) the membrane, and one for the current density between the electrolyte and the electrode. Note these current densities are per the geometric cross sectional areas of the cell and not per the actual surface area of the electrode. The associated ohmic and kinetic drop is also assigned to each electrode section. They are also treated as having their own $S O C_{r x n}$, as the assumption of uniform current distribution is no longer being used. 


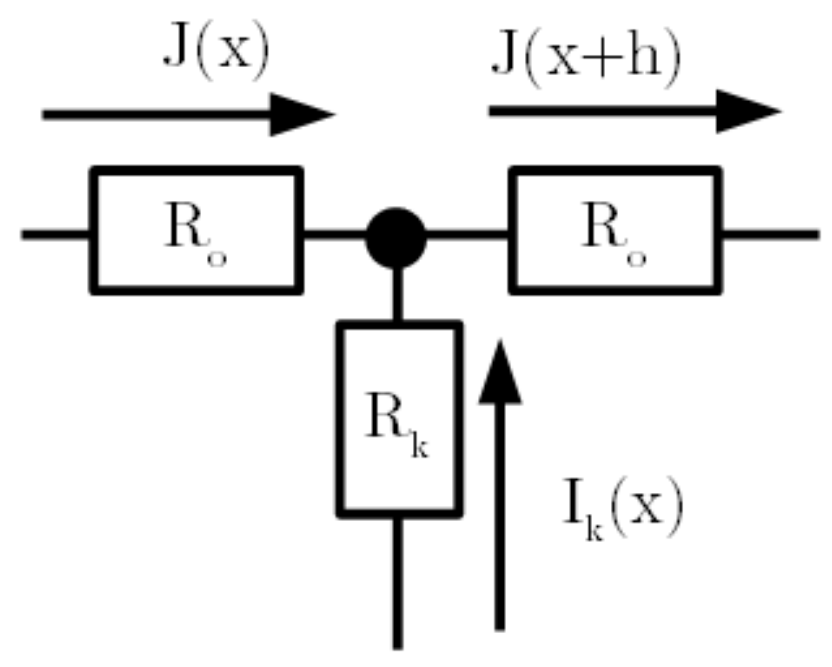

Figure 4.3. Current conservation at a single junction

This treatment can be drawn as a series of resistors, as shown in Figure 4.2. The vertical resistors represent the charge transfer resistance and drop due to the kinetics term, while the horizontal resistors represent the ohmic drop. It is assumed that the ohmic drop due to current within the felt electrode is negligible due to its high conductivity (11 orders of magnitude greater), so a resistor is not drawn. $J_{t o t}$ refers to the total current density that enters the system, and exits at the membrane.

A single junction is given in Figure 4.3, with $J$ referring to the ionic current density traveling towards the membrane and I referring to the current density added to the electrolyte. In order to conserve current, the following condition must be met:

$$
J(x+h)=J(x)+I(x)
$$

This equation can be rearranged, 


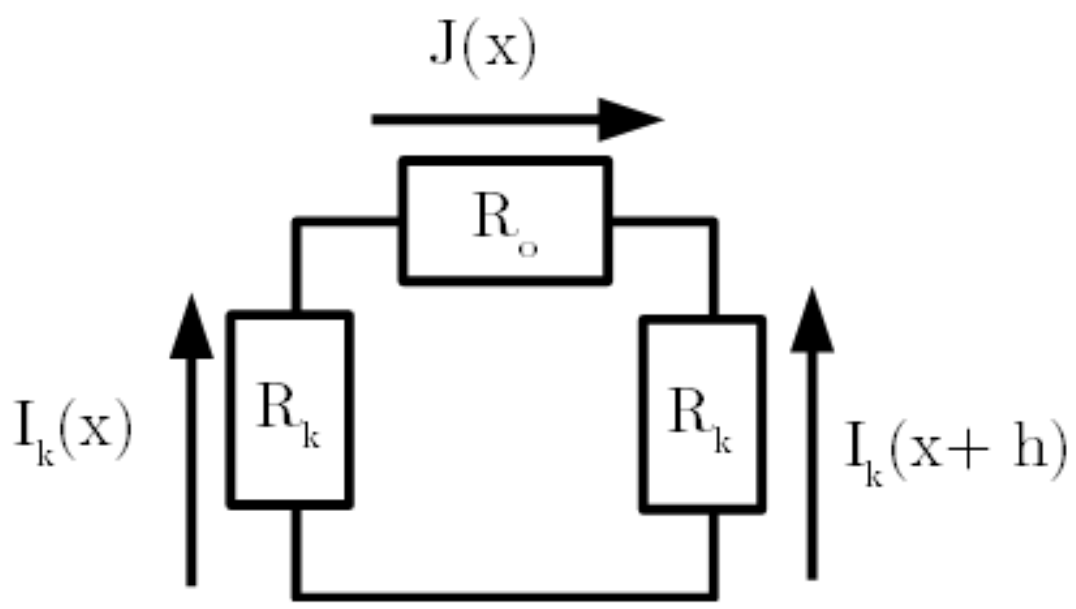

Figure 4.4. A loop of resistors for which Kirchhoff's loop law can be applied to conserve voltage.

$$
\frac{J(x+h)-J(x)}{h}=\frac{I(x)}{h}
$$

As $\mathrm{h}$ goes to zero, the left hand side becomes the derivative of $J$ with respect to $\mathrm{x}$. The right hand side goes to the electrode current density per unit length, which is a volumetric current density and is represented by $i$. This can also be understood as the reaction rate per volume. The equation becomes:

$$
\frac{d J}{d x}=i
$$

This gives us one of our fundamental equations that conserves the current.

Next we must conserve voltage, and look at a loop of resistors given in Figure 4.4. Due to Kirchhoff's loop law, going around one of these loops must result in a total potential change of zero. Therefore by taking into account the ohmic and kinetic drops 
while going around one of these loops,

$$
\eta_{k}(x+h)-\eta_{k}(x)-J(x) \rho h=0
$$

Rearranging:

$$
\frac{\eta_{k}(x+h)-\eta_{k}(x)}{h}=J(x) \rho
$$

Again, h goes to zero resulting in the following equation:

$$
\frac{d \eta}{d x}=J \rho
$$

As $h$, the section thickness goes to zero (and the number of sections goes to infinity), we obtain the Equations 4.3 and 4.6. These are the fundamental equations for solving the current distribution. This can be used to calculate the performance of the electrode. The boundary conditions are that $\mathbf{J}$ must be the maximum applied current density at the membrane surface as this is what enters the membrane, and zero at the current collector (as there is no ionic current density in the current collector).

$$
J(0)=0
$$

$$
J\left(t_{\text {electrode }}\right)=J_{\text {tot }}
$$


The next step is to rewrite the kinetic drop in terms of the local volumetric current density. For a reacting thickness $\mathrm{h}$, the term $\frac{j}{h}$ goes to the volumetric current density as $\mathrm{t}$ goes to zero.

$$
\eta_{a, k}=\frac{R T}{F} \operatorname{asinh}\left(\frac{j}{2 a F k_{a}} \frac{1}{h \sqrt{V_{I I} V_{I I I}}}\right)
$$

The two concentrations on the bottom are themselves functions of the volumetric current density, as it governs the SOC of reaction.

$$
\begin{gathered}
S O C_{r x n}=S O C-\triangle S O C \\
\Delta S O C=\frac{I}{Q C F}+\frac{1}{F(a / \epsilon) k_{m}} \frac{j}{h}
\end{gathered}
$$

Note that $\mathrm{Q}$ is only the volumetric flow rate going through the thickness $\mathrm{h}$, and $\mathrm{I}$ is the current going through the thickness $\mathrm{h}$. Therefore the utilization portion of the $\triangle S O C$ can be rewritten as:

$$
\Delta S O C=\frac{i A_{\text {electrode }} h}{Q \frac{h}{t_{\text {electrode }}} C F}+\frac{1}{F(a / \epsilon) k_{m}} \frac{j}{h}
$$

Where $A_{\text {electrode }}$ is the cross sectional area of the electrode (and also the volume being analyzed). Rearranging, we find the $\triangle S O C$

$$
\Delta S O C=i \frac{t_{\text {electrode }} A_{\text {electrode }}}{Q C F}+\frac{1}{F(a / \epsilon) k_{m}} i
$$


This gives us a function for our concentration terms based on the volumetric current density.

$$
\begin{gathered}
V_{I I}=C S O C-\left(\frac{1}{F(a / \epsilon) k_{m}}+C \frac{A_{\text {electrode }} t_{\text {electrode }}}{Q C F}\right) i \\
V_{I I I}=C-C S O C+\left(\frac{1}{F(a / \epsilon) k_{m}}+C \frac{A_{\text {electrode }} t_{\text {electrode }}}{Q C F}\right) i
\end{gathered}
$$

Using constants to simplify,

$$
\begin{gathered}
V_{I I}=C_{I I}-i C_{T} \\
V_{I I I}=C_{I I I}+i C_{T}
\end{gathered}
$$

Plugging this product into Equation 4.9 obtains the kinetic drop as a function of volumetric current density:

$$
\eta=\frac{R T}{F} \operatorname{asinh}\left(\frac{1}{2 a F k_{1}} \frac{i}{\sqrt{\left(C_{I I}-i C_{T}\right)\left(C_{I I I}+i C_{T}\right)}}\right)
$$

This equation is rearranged

$$
\eta=\frac{R T}{F} \operatorname{asinh}\left(\frac{1}{2 a F k_{1} C_{T}} \frac{i}{\sqrt{\left(\frac{C_{I I}}{C_{T}}-i\right)\left(\frac{C_{I I I}}{C_{T}}+i\right)}}\right)
$$


Finally this gives us the following simplified equation for the kinetic drop as a function of volumetric current density:

$$
\eta=A \operatorname{asinh}\left(\frac{B i}{\sqrt{(D-i)(G+i)}}\right)
$$

We can conclude from this the requirement that i must lie between 0 and $\mathrm{D}$, to prevent this overpotential going to infinity. Going forward, any functions of $i$ are analyzed staying within this domain.

Next, we go back to the fundamental Equation 4.6.Using the chain rule to expand the left hand side results in:

$$
\frac{d \eta}{d i} \frac{d i}{d J} \frac{d J}{d x}=J \rho
$$

Note that $\frac{d J}{d x}=i$, this transforms the differential equation relating $\mathbf{J}$ and $\mathbf{i}$ without $\mathbf{x}$, producing an autonomous equation.

$$
\frac{d \eta}{d i} \frac{d i}{d J} i=J \rho
$$

This equation can be separated, in order to be integrated

$$
i \frac{d \eta}{d i} d i=J \rho d J
$$

Both sides are integrated, with constant of integration $\mathrm{K}$. The left side is integrated using integration by parts to simplify. 


$$
i \eta-\int \eta d i=\frac{\rho}{2} J^{2}+K
$$

It is possible to integrate the left hand side analytically, leading to the unwieldy expression:

$$
\begin{gathered}
\frac{\rho}{2} J^{2}+C=\frac{A}{2}\left(D \log \left(D\left(2 B \alpha+2 B^{2} i+G-i\right)+D^{2}-G i\right)+(G-D) \log \left(2 \alpha+\frac{\left(2 B^{2} i+D-G\right)}{B}\right)\right. \\
+\frac{A}{2}\left((G-D) \log \left(2 \alpha+\frac{\left(2 B^{2} i+D-G\right)}{B}\right)+G \log \left(G\left(2 B \alpha-2 B^{2} i+G+i\right)+D(G+i)\right)\right) \\
-\frac{A}{2}(D \log (D-i)+G \log (G+i))
\end{gathered}
$$

Where within Equation 4.25, the following substitution is made:

$$
\alpha=\sqrt{i\left(B^{2} i-G\right)+D(G+i)}
$$

This gives an equation relating $\mathrm{i}$ and $\mathrm{J}$, which is enough to numerically solve for the current distribution. However it requires some approximation in order to continue. Going forward, any constants added on this side will be ignored, as they can be absorbed into the constant on the right hand side.

Noting that this equation blows up as $\mathrm{i}$ approaches its maximum value of $\mathrm{D}$, and 
noticing that the $\ln (D-i)$ part is the largest part of the equation, Equation 4.25 is broken into two parts

$$
\frac{\rho}{2} J^{2}+K=-\frac{A D}{2} \log \left(1-\frac{i}{D}\right)+\frac{A}{2} g(i)
$$

With $g(i)$ given by

$$
\begin{aligned}
g(i)=D & \log \left(D\left(2 B \alpha+2 B^{2} i+G-i\right)+D^{2}-G i\right)+(G-D) \log \left(2 \alpha+\frac{\left(2 B^{2} i+D-G\right)}{B}\right) \\
& +G \log \left(G\left(2 B \alpha-2 B^{2} i+G+i\right)+D(G+i)\right)-G \log (G+i)
\end{aligned}
$$

The $\ln \left(1-\frac{i}{D}\right)$ portion is the largest part of the function. Modeling the rest of the function to fit in with this is attractive, as it gives us a single function for which we can solve for $\mathrm{i}$. We also want to maintain the behavior that this function blows up at $i=D$, so that going forward $\mathrm{i}$ will never be equal to or greater than $\mathrm{D}$.

Therefore we want to approximate $g(i)$ in terms of $\log \left(1-\frac{i}{D}\right)$. Using the approximation for this expression when $\mathrm{i}$ is equal to half of its maximum value, or $\frac{D}{2}$ gives a good approximation of all possible values of $i$.

$$
\ln \left(1-\frac{i}{D}\right) \approx-\frac{2 i}{D}+K
$$

Next $g(i)$ is turned into a linear approximation (noting that only the slope is required, 
as the intercept is absorbed into the constant on the other side of the equation) and then using Equation 4.29 turned into a logarithmic approximation:

$$
g(i) \approx \frac{g(D)-g(0)}{D} i \approx-\frac{1}{2}(g(D)-g(0)) \ln \left(1-\frac{i}{D}\right)
$$

Putting all together with this new approximation we obtain

$$
\frac{\rho}{2} J^{2}+K=-\frac{A}{2}\left(D-\frac{g(D)-g(0)}{2}\right) \ln \left(1-\frac{i}{D}\right)
$$

Rearranging results in:

$$
K-\frac{\rho}{A(D-(g(D)-g(0)) / 2)} J^{2}=\ln \left(1-\frac{i}{D}\right)
$$

For simplicity, another constant $\mathrm{m}$ is introduced. This $\mathrm{m}$ can be interpreted as the ratio between the ohmic resistance and the charge transfer resistance.

$$
K-m J^{2}=\ln \left(1-\frac{i}{D}\right)
$$

This equation is solved in terms of $\mathrm{i}$ :

$$
i=D\left(1-K \exp \left(-m J^{2}\right)\right)
$$

This equation for i behaves as we would expect, with the volumetric current density and $\mathbf{J}$ increasing together in the direction towards the membrane, and as the right hand 
side cannot be greater than $\mathrm{D}$, this captures the limitations on $\mathrm{i}$.

As we know the maximum value of $\mathrm{J}$, this means we can calculate the maximum value of $\mathrm{i}$ at the membrane as long as we can solve for K. Going forward, an analytical method is compared to solve for $\mathrm{K}$ and the maximum value of $\mathrm{i}$. This is compared to a Runga-Kutta method that was used to solve for $\mathrm{K}$ and the maximum value of $\mathrm{i}$ from Equation 4.25 in order to gauge the error from the approximations used to get to this point.

The equation is written so that it is separable, and ready to be integrated.

$$
d x=\frac{1}{D} \frac{1}{\left(1-K \exp \left(-m J^{2}\right)\right)} d J
$$

The left hand side is integrated from 0 to the entire thickness of the electrode, $t_{\text {electrode }}$ as the right hand side is integrated over $\mathrm{J}$ which goes from 0 to $J_{\text {tot }}$

$$
t_{\text {electrode }}=\int_{0}^{J_{\text {tot }}} \frac{1}{D} \frac{1}{\left(1-K \exp \left(-m J^{2}\right)\right)} d J
$$

This gives the criteria by which we can solve for $\mathrm{K}$. Unfortunately this equation is not solvable in terms of elementary functions, and therefore must be solved numerically.

This integral can be rewritten with some constants to give a more general integral to solve.

$$
y=\frac{J}{J_{t o t}}
$$




$$
\begin{gathered}
\frac{D t_{\text {electrode }}}{J_{\text {tot }}}=\int_{0}^{1} \frac{1}{\left(1-K \exp \left(-m J_{\text {tot }}^{2} y^{2}\right)\right)} d y \\
r=\int_{0}^{1} \frac{1}{\left(1-K \exp \left(-b y^{2}\right)\right)} d y
\end{gathered}
$$

By rewriting this integral generally, we can analyze the error of any approximation scheme we come up with as a function of $r$ and $b$. The physical significance of $r$ can be seen to simply be the inverse of the ratio of the average volumetric current density $\frac{J_{t o t}}{t_{\text {electrode }}}$ to the highest possible current density D. The constant $\mathrm{b}$ is related to the ratio of ohmic losses to kinetic losses, with b increasing for high current and low conductivity, and decreasing with improved kinetics and higher SOCs.

The first approximation is using the trapezoid method to integrate the right hand side using the values at the minimum and maximum values of $\mathrm{J}$.

$$
r \approx \frac{1}{2}\left(\frac{1}{(1-K)}+\frac{1}{(1-K \exp (-b))}\right)
$$

Rearranging gives the quadratic equation that can be solved for $\mathrm{K}$ :

$$
K_{0}^{2}(2 r \exp (-b))+K_{0}(1+\exp (-b)-2 r-2 r \exp (-b))+(2 r-2)=0
$$

Solving this quadratic equation gives a first approximation for $\mathrm{K}$, or $K_{0}$. This approximation by itself is fairly good in certain regions, with the error in volumetric 


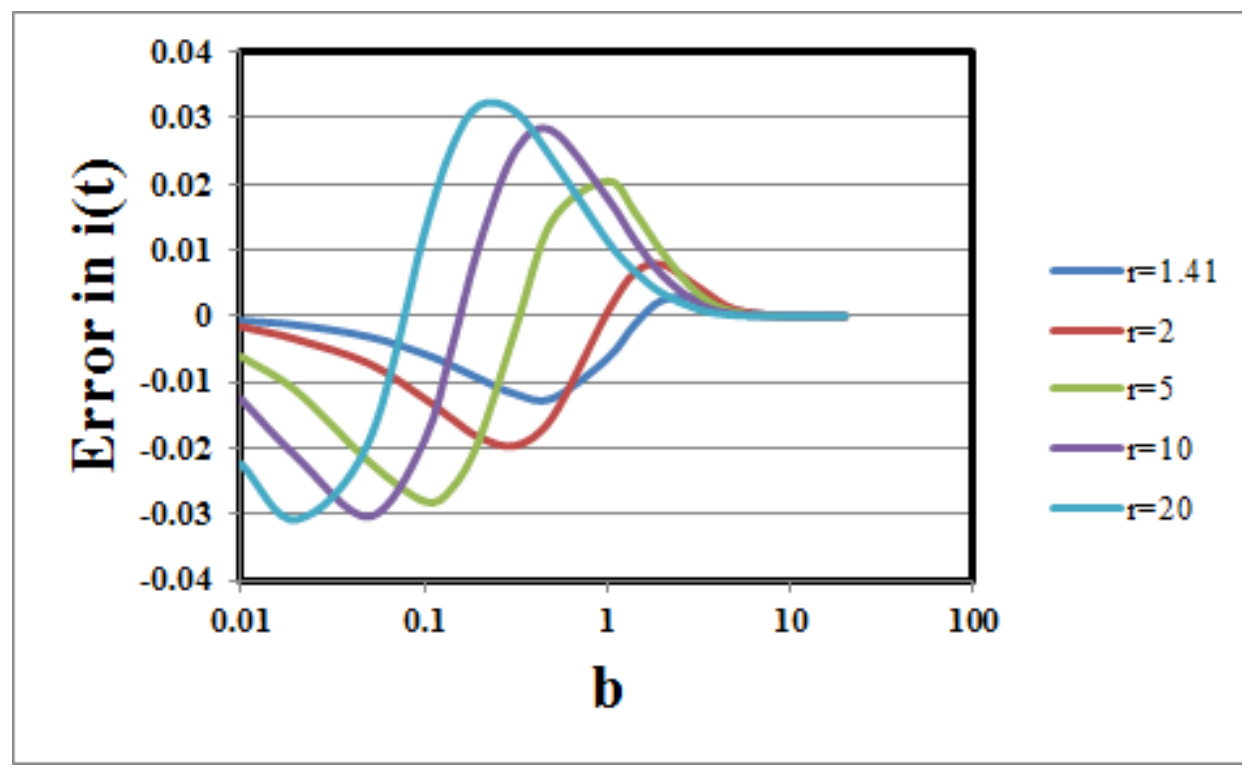

Figure 4.5. Error in volumetric current density calculated using first approximation

current density at the membrane at various values of $r$ as a function of $b$ is given in Figure 4.5 .

To solve for $\mathrm{K}$ more accurately, we must come up with a way to more precisely approximate the integral in Equation 4.39. The following substitution is made:

$$
u=\exp \left(b y^{2}\right)
$$

This results in 4.39 being transformed into the following integral:

$$
r=\frac{1}{2 \sqrt{b}} \int_{1}^{\exp b} \frac{1}{(u-K)} \frac{1}{\sqrt{\ln u}} d u
$$

Now we can approximate theln $u$ term around $u=1$. As the function being integrated blows up around $u=1$, this is where most of the area to integrate is located. The 
approximation of the $\ln u$ term is very accurate near $u=1$, and when $u$ becomes much larger and the approximation is less valid, the function becomes very small due to the $\frac{1}{u-K}$ term. Therefore this approximation ensures minimization of error. The integral becomes:

$$
r \approx \frac{1}{2 \sqrt{b}} \int_{1}^{\exp b} \frac{1}{(u-K)} \frac{1}{\sqrt{u-1}} d u
$$

This integral can be analytically evaluated, resulting in:

$$
\sqrt{b} r=\frac{1}{\sqrt{1-K}} \tan ^{-1} \frac{\sqrt{\exp b-1}}{\sqrt{1-K}}
$$

Now we can solve for K. To make this easier, we make the following definition:

$$
g=\frac{1}{\sqrt{1-K}}
$$

By this definition, we also find the approximate value of $g$ based on the first approximation of $K$.

$$
g_{0}=\frac{1}{\sqrt{1-K_{0}}}
$$

The Equation 4.45becomes:

$$
\sqrt{b} r=g \tan ^{-1}(g \sqrt{\exp b-1})
$$

This equation is approximated around $g_{0}$ to obtain a linear function, as $\mathrm{g}$ should be 


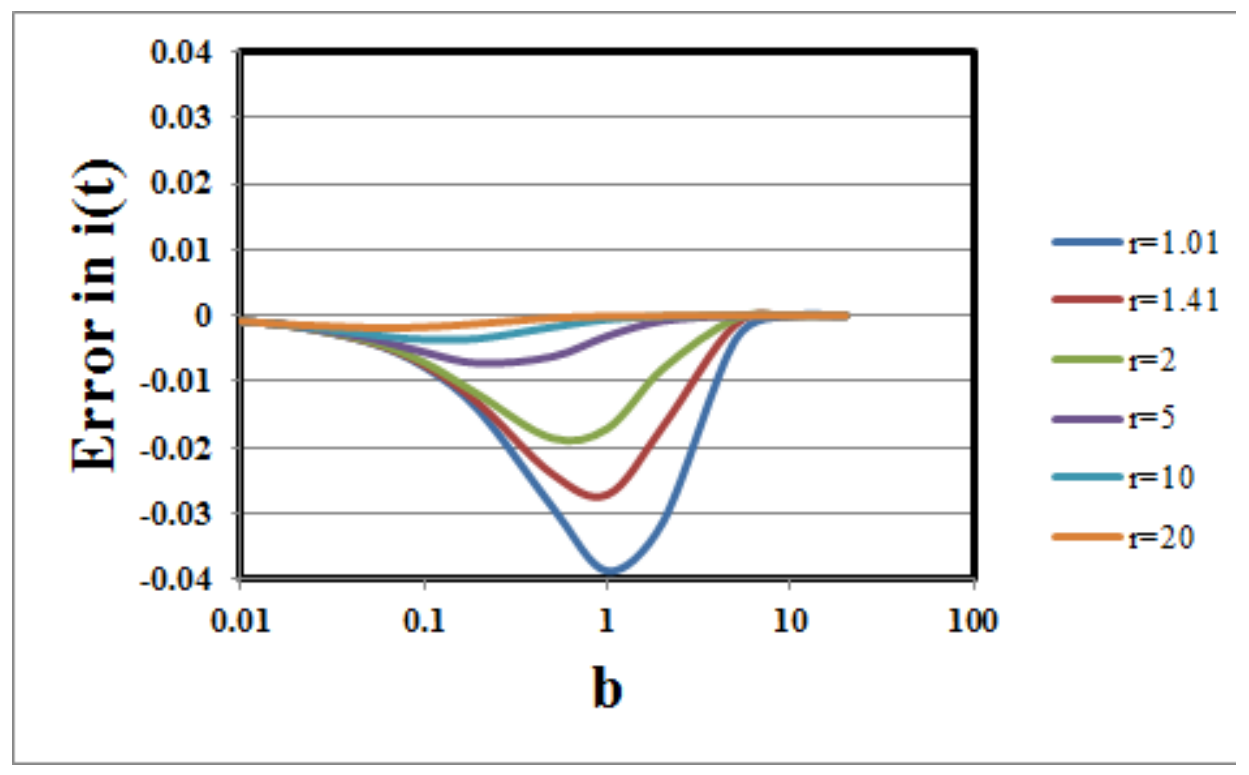

Figure 4.6. Error in volumetric current density calculated using integration approximation method

close to $g_{0}$

$\sqrt{b} r \approx g_{0} \tan ^{-1}\left(g_{0} \sqrt{\exp b-1}\right)+\left(g-g_{0}\right)\left(\frac{g_{0} \sqrt{\exp b-1}}{g_{0} \exp b-g_{0}+1}+\tan ^{-1}\left(g_{0} \sqrt{\exp b-1}\right)\right)$

This allows us to solve for $g$, and therefore for $\mathrm{K}$.

$$
\begin{gathered}
\frac{\sqrt{b} r+\left(\frac{g_{0}^{2} \sqrt{\exp b-1}}{g_{0} \exp b-g_{0}+1}\right)}{\left(\frac{g_{0} \sqrt{\exp b-1}}{g_{0} \exp b-g_{0}+1}+\tan ^{-1}\left(g_{0} \sqrt{\exp b-1}\right)\right)}=g \\
K=1-\frac{1}{g^{2}}
\end{gathered}
$$

After obtaining $\mathrm{K}$, it is fairly straight forward to find the volumetric current density at the membrane. 


$$
i_{\max }=D\left(1-K \exp \left(-m J_{\text {tot }}^{2}\right)\right)
$$

In order to get to this point, quite a few approximations were made. First the logarithmic approximation to arrive at Equation 4.31, and then solving for $K$. In order to evaluate the error inherent in this approximation scheme, various current distributions were calculated by evaluating the exact solution in Equation 4.25. This equation was evaluated numerically using the Runga-Kutta method, with $\mathrm{K}$ being set to satisfy the boundary conditions by using Newton-Rhapson iteration. This was used to calculate the error in volumetric current density at the membrane.

The error as a function of $b$ for various $r$ values is presented in Figure 4.6. It can be seen that for most values of $\mathrm{r}$ and $\mathrm{b}$, the error is very small, with one exception when $b$ and $r$ are both very close to 1 . The physical meaning of this specific scenario is that the ohmic and charge transfer resistances are approximately equal, and the average volumetric current density is very close to the maximum possible current density. This means the performance is very low, with the $S O C_{r x n}$ collapsing almost to zero - with the performance collapsing, this likely isn't an area of interest for operating the battery. For the $\mathrm{V}-\mathrm{V}$ system, $\mathrm{r}$ vs $\mathrm{b}$ has been plotted for all current densities and flow rates in Figure 4.9, showing that for this system, when $\mathrm{b}$ is near $1, \mathrm{r}$ is quite high, meaning the losses are quite low and having error is more acceptable. Outside of this specific area, the error quickly falls, becoming negligible unless $b$ is within half an order of magnitude of 1 .

The current distribution can be solved for now, although it is not used in the 
performance model it may be of some interest for future work. From Equation,

$$
x=\frac{J_{t o t}}{D} \frac{1}{2 \sqrt{b}} \int_{1}^{\exp \left(m J^{2}\right)} \frac{1}{(u-K)} \frac{1}{\sqrt{\ln u}} d u
$$

Integrating similar to before with the approximation for logarithmic term we obtain:

$$
x=\frac{1}{D \sqrt{m}} \frac{1}{\sqrt{1-K}} \tan ^{-1} \frac{\sqrt{\exp \left(m J^{2}\right)-1}}{\sqrt{1-K}}
$$

Solving for $\mathrm{J}$ allows us to write this in a normalized form:

$$
\frac{J}{J_{\text {tot }}}=\sqrt{\frac{\ln \left((1-K) \tan ^{2}\left(r \sqrt{b} \sqrt{1-K} \frac{x}{t_{\text {electrode }}}\right)+1\right)}{b}}
$$

Taking the derivative of this gives us the function for the volumetric current density:

$$
\frac{i}{D}=\frac{(1-K) \tan \left(r \sqrt{b} \sqrt{1-K} \frac{x}{t_{\text {electrode }}}\right)}{\sqrt{2 \ln \left(\sec \left(r \sqrt{b} \sqrt{1-K} \frac{x}{t_{\text {electrode }}}\right)\right)}}
$$

It can be seen that $r, b$, and $\mathrm{K}$ which is a function of $r$ and $b$ affect the current distribution. Therefore there should be one characteristic quantity built out of these 3 quantities that govern the current distribution. To find this quantity, the distribution was found for a given $r$ and $b$. Then, after changing $b$, the $r$ corresponding to the same current distribution was found. Plotting $r-1$ vs $b$ for 3 of these current distributions is given in Figure 4.7. It can be seen that a given current distribution as long as $(r-1) b$ equals the characteristic value associated with this current distribution hence $(r-1) b$ is the 


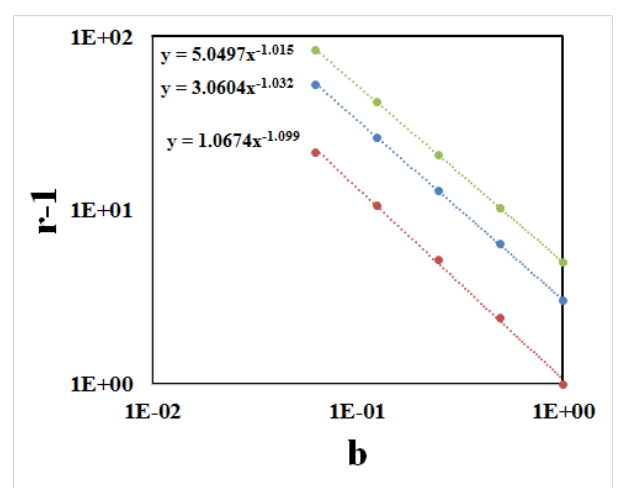

Figure 4.7. Curves for $r-1$ vs $b$ giving the same current distribution

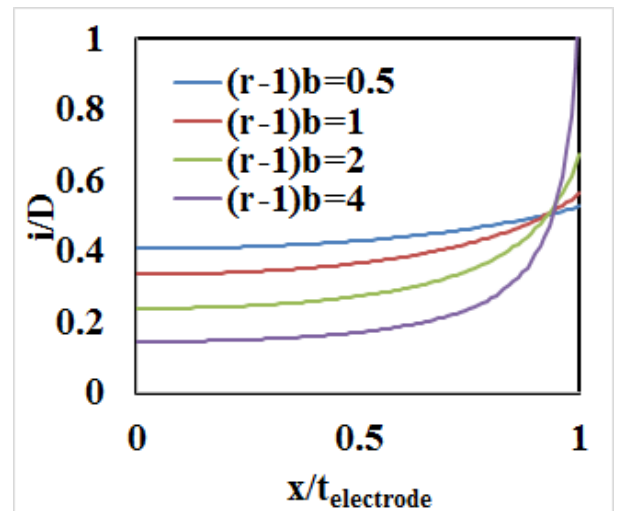

Figure 4.8. Volumetric current distributions for various $(r-1) b$

characteristic value.

Therefore the value $(r-1) b$ can be thought of as the ratio between ohmic and charge transfer resistances. When this quantity is large, ohmic losses are dominating, and the volumetric current density is concentrated at the membrane in order to bypass most of the ohmic resistance. When this quantity is small, charge transfer losses are dominating, and the volumetric current density is more uniform in order to reduce these losses. An example of various curves for different $(r-1) b$ values is given in Figure 4.8

We can utilize our knowledge of the characteristic value in order to come up with a very fast approximation. Current distributions were found for various values of $r$ and $b$, 


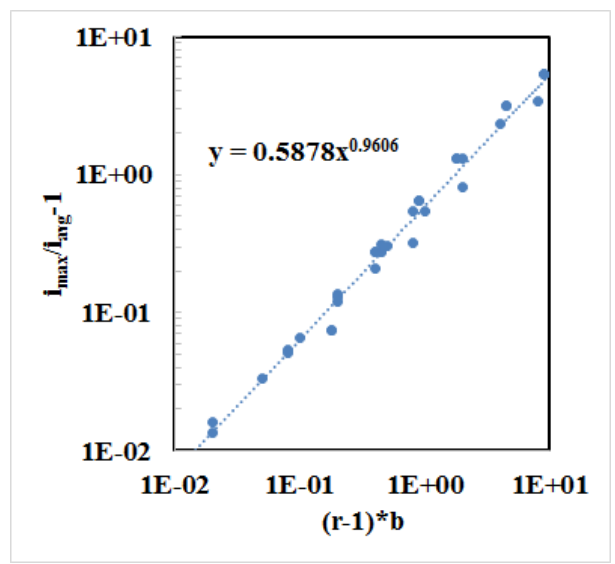

Figure 4.9. $\frac{i_{\max }}{i_{a v g}}-1$ plotted for various values of $\mathrm{r}$ and $\mathrm{b}$

and the quantity $\frac{i_{\max }}{i_{\text {avg }}}-1$ was plotted vs $(r-1) b$ in Figure 4.9.The quantity $\frac{i_{\max }}{i_{\text {avg }}}-1$ is essentially the uniformity of the current distribution, with this quantity being very small when the current distribution is very spread out and $i_{\text {max }} \approx i_{\text {avg }}$, and large when the current distribution is concentrated at the membrane and $i_{m a x}$ is much bigger than $i_{\text {avg }}$.

The relationship is almost linear, but can be more accurately modeled with a power curve. Therefore $i_{\max }$ can be approximated with the following:

$$
i_{\text {max }} \approx i_{\text {avg }}\left(1+0.5878[(r-1) b]^{0.9606}\right)
$$

This function could be useful for a very rapid way of calculating the performance, but has the drawback of not preventing $i_{\max }$ from going to D. It is more useful for understanding how the current distribution uniformity varies, which could be useful in future work. 


\section{B. Validation}

Using the same approach as with the effective thickness model, the current distribution model was matched to the same 21 cell stack data, and is plotted in Figures 4.10, 4.11, and 4.12. It can be seen that in general, the model is a good fit, but tends to underestimate performance at high and low SOC, and overestimate in the middle of the SOC range. It also overestimates the effect of flow rate, with the model underestimating performance more for low flow rates, and overestimating more for high flow rate.

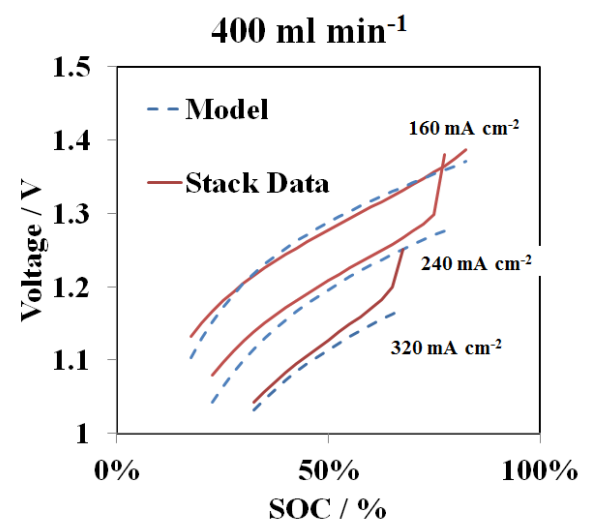

Figure 4.10. Current Distribution Model vs Stack Data for $400 \mathrm{ml} \mathrm{min}{ }^{-1}$

A summary of all flow rates and current densities is presented in Figure 4.13, demonstrating the good fit of the model to stack data. The root mean square error for all scenarios is $21 \mathrm{mV}$.

Another set of experimental data was obtained for a novel, proprietary electrode with a much thinner electrode thickness and drastically increased surface area, which was also modeled with the current distribution model. Again this cell was operated at 3 different current densities, with 3 different flow rates for each for a total of 9 different operating 


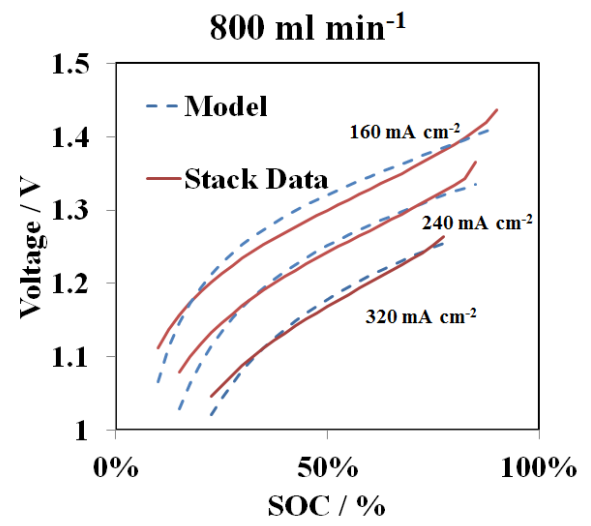

Figure 4.11. Current Distribution Model vs Stack Data for $800 \mathrm{ml} \mathrm{min}^{-1}$

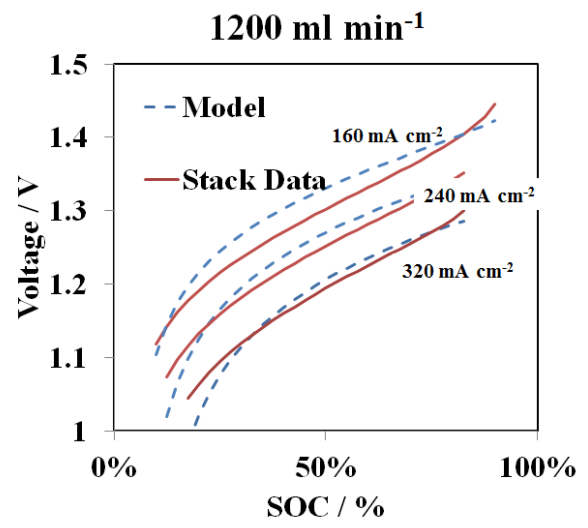

Figure 4.12. Current Distribution Model vs Stack Data for $1200 \mathrm{ml} \mathrm{min}{ }^{-1}$

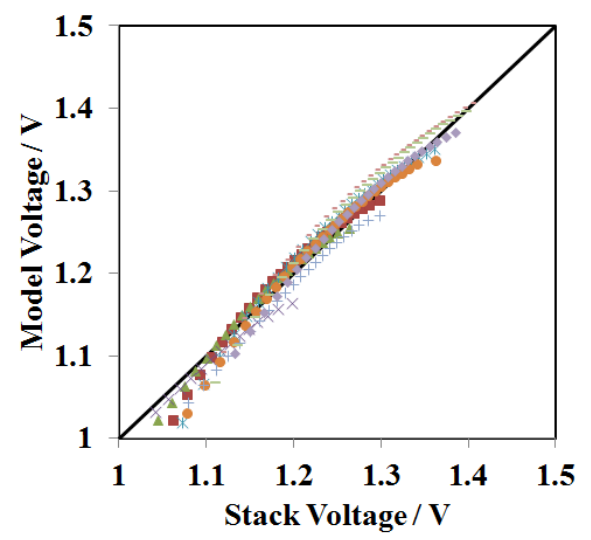

Figure 4.13. Current Distribution Model vs Stack Data for all scenarios 


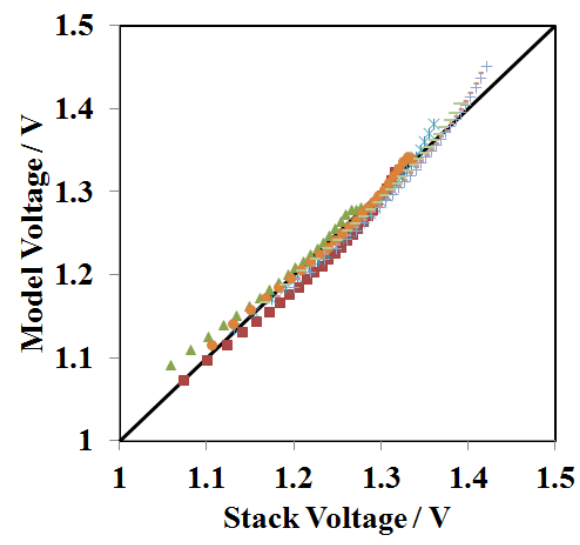

Figure 4.14. Current Distribution Model vs Stack Data for Novel Electrode Material

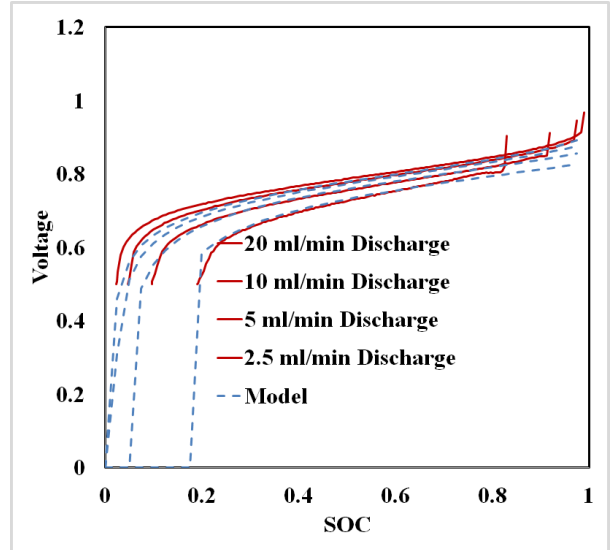

Figure 4.15. Current Distribution Model vs Stack Data for Fe-V Single Cell

conditions. These results are given in Figure 4.14, demonstrating the model's ability to fit well to a scenario with different electrochemical parameters and electrode thickness. This implies the model can be used at least for $\mathrm{V}-\mathrm{V}$ systems without requiring empirical fitting.

The model was also applied to another chemistry, a single cell Fe-V battery. Not as much data was available for this single cell, the model results vs data are given in Figure 4.15. 


\section{Comparison with Effective Thickness Model}

In general, the current distribution model fits the performance data obtained less accurately than the effective thickness model, with the root mean square error squared coming to $16 \mathrm{mV}$ for the effective thickness model and $21 \mathrm{mV}$ for the current distribution model. In general the current distribution over predicts at the middle of the SOC range and under predicts at low SOC. There is still reason to use the current distribution model, however. As the physical principles behind the current distribution model are sound, it may apply to a wider variety of chemistries. It should more accurately capture trends from varying parameters such as kinetic rate constant, specific surface area, and electrode thickness. It could also be possible to perturb the solution obtained for the current distribution model in order to include other physical phenomena and make the model more accurate across the SOC range.

On top of that, the current distribution model requires less computation time than the effective thickness model, making it easier to utilize in the battery optimization routine. Also as discussed previously, the effective thickness model assumptions may not work for optimizing electrode thickness, due to the assumption of uniform current distribution.

All of these benefits overshadow the fact that the RMS error is approximately $30 \%$ greater ( $21 \mathrm{vs} 16 \mathrm{mV}$ ) for the current distribution model for the data investigated. There is still room for improvement in the current distribution model by taking more phenomena into account, and the models are both fairly accurate in the first place. 


\section{APPLICATION TO NON-AQUEOUS SYSTEM}

While most research into RFBs has focused on aqueous systems due to high electrolyte conductivity, there has been interest in researching non-aqueous RFB systems. Nonaqueous systems have significantly lower conductivity, and thus cannot support high current density as high as aqueous RFB systems. However, they can have a much higher voltage due to a high $E_{0}$, and the possibility of using divalent active species. The ability to use divalent active species would effectively double energy density and half energy cost. Both of these factors make non-aqueous systems possible candidates for high energy applications.

Not much modeling work has been done on non-aqueous systems, with the closest attempt at cost modeling a non-aqueous system being performed by Darling et al [5]. A much simplified physical model was used for this model, with the voltage being assumed to be linear with current, and the power being constant across the SOC range. This does not provide guidance towards developing cost effective non-aqueous systems.

Therefore the non-aqueous case was modeled to investigate its practicality and to provide guidance on developing the systems on where more improvements must be made. The effective thickness model was used to determine performance, as this analysis was performed after developing the effective thickness model but before the current distribution model.

\section{A. System parameters}

The electrolyte ionic conductivity used was significantly lower than aqueous systems, using a value of $1 \mathrm{~S} \mathrm{~m}^{-1}$, and the standard cell potential used was $1.96 \mathrm{~V}$ [1]. The 
kinetic rate constant was measured at MIT as $1.4 \times 10^{-5} \mathrm{~m} \mathrm{~s}^{-1}$ for 2,5-Di-tert-butyl-1,4bis(2-methoxyethoxy)benzene (DBBB), the positive active species and assumed to be the same for the negative active species [2].

A conservative value for the active species concentration of $1 \mathrm{M}$ was used, and a more optimistic concentration of $2 \mathrm{M}$. Scenarios of monovalent and divalent active species were also investigated to determine how this affects system cost.

Each of these cost analysis were done for various energy to power ratios, to determine which applications the non-aqueous system would be appropriate for.

For modeling the pressure drop, the viscosity was taken to be $2.4 \mathrm{cP}$ [7], the same order of magnitude as aqueous systems but reducing by a factor of 2 at $60 \mathrm{C}$.

The same cost parameters for stack components was used as from the previous V-V system modeling, as the same stack components would be used. The only cost parameter difference is electrolyte cost. For the non aqueous system it was taken to be $18 \$ / \mathrm{kg}$ for present case, going to an optimistic value of $5 \$ / \mathrm{kg}$. This price was based on bulk Li-ion battery electrolyte, $\mathrm{LiPF}_{6}$ salt, and EC and DMC pricing obtained from Alibaba. Note that unlike the V-V chemistry where the active species is the dominating cost, and hence changing concentration does not directly change the $\$ / \mathrm{Ah}$, in this non aqueous chemistry the dominating cost is the electrolyte solvent. Hence for the non aqueous chemistry increasing the concentration directly decreases electrolyte cost.

\section{B. Results}

Whenever a cost for the non-aqueous system was calculated, the operating parameters were optimized to provide the lowest cost. The electrode thickness was also optimized 


\begin{tabular}{|c|c|c|}
\hline $\mathrm{E} / \mathrm{P} / \mathrm{h}$ & 12 & 0.1 \\
\hline Total Cost / \$ kWh ${ }^{-1}$ & 11100 & $\overline{5950}$ \\
\hline Energy Cost $/ \$ \mathrm{kWh}^{-1}$ & 1055 & 1440 \\
\hline Power Cost / \$ kW $\mathrm{kW}^{-1}$ & 720 & 455 \\
\hline Current Density / $\mathrm{mA} \mathrm{cm}^{-2}$ & 165 & 240 \\
\hline Flow Rate $/ \mathrm{ml} \mathrm{min}^{-1} \mathrm{~A}^{-1}$ & 13.7 & 5.9 \\
\hline SOC range $/ \%$ & 89 & 68 \\
\hline Area $/ \mathrm{cm}^{-2}$ & 3010 & 1480 \\
\hline Electrode Thickness / mm & 0.19 & 0.17 \\
\hline
\end{tabular}

OPTIMIZED PARAMETERS FOR BASELINE NONAQEUOUS SYSTEM

to provide the lowest cost, which is particularly important for this system. The low conductivity of the non-aqueous system forces a low effective thickness, meaning that the electrode thickness must also be low in order not to waste energy pumping nonreacting electrolyte.

modeling was done for both conventional cell design and a cell with interdigitated flow. Interdigitated flow has several flow channels within the felt, which reduce the pressure drop significantly due to a lower average distance traveled by the electrolyte. This factor by which the pressure drop is reduced is approximately equal to the number of flow channel. The decrease in cost due to this decrease in pressure drop is taken into account in the cost calculations. Note that the inter-channel separation used for this analysis was $2 \mathrm{~cm}$ based on mechanical considerations (a lower channel separation results in lower pressure drop)

There were 3 cost scenarios used for each component to show the amount by which the cost could vary due to the components - these are referred to as present, near-term, and optimistic.

Figures 5.1 and 5.2 show the costs for the 0.1 -h case and the 12-h case respectively. 


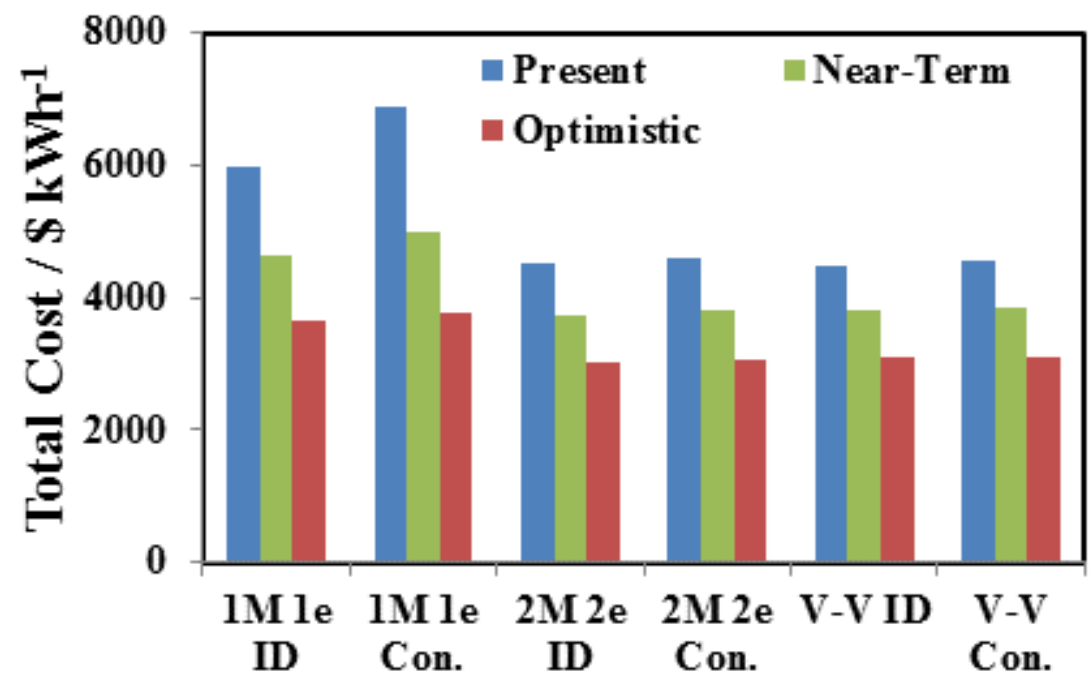

Figure 5.1. Costs for various chemistries for 0.1-h case

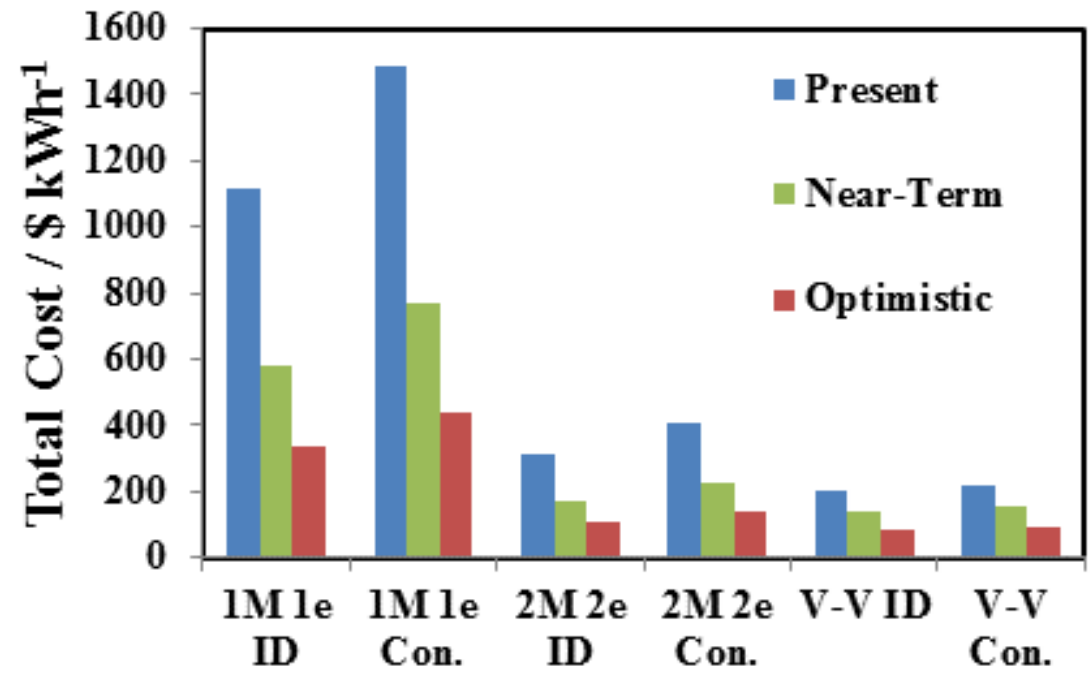

Figure 5.2. Costs for various chemistries for 12-h case

The present costs for a $1 \mathrm{M}$ non-aqueous system is 7000 and $6000 \$ \mathrm{kWh}^{-1}$ for $\mathrm{E} / \mathrm{P}=$ 0.1-h for conventional and interdigitated flow respectively, while the corresponding cost for a $2 \mathrm{M}$ all vanadium system are $4500 \$ \mathrm{kWh}^{-1}$ for both flow patterns. At $\mathrm{E} / \mathrm{P}$ of $12-\mathrm{h}$, the non-aqueous system costs of $1500 \$ \mathrm{kWh}^{-1}$ and $1120 \$ \mathrm{kWh}^{-1}$ for conventional and 


\begin{tabular}{|c|c|c|}
\hline $\mathrm{E} / \mathrm{P} / \mathrm{h}$ & 12 & 0.1 \\
\hline Total Cost / \$ kWh ${ }^{-1}$ & 310 & $\overline{4500}$ \\
\hline Energy Cost $/ \$ \mathrm{kWh}^{-1}$ & 255 & 445 \\
\hline Power Cost / \$ kW $\mathrm{kW}^{-1}$ & 660 & 410 \\
\hline Current Density / $\mathrm{mA} \mathrm{cm}^{-2}$ & 148 & 240 \\
\hline Flow Rate $/ \mathrm{ml} \mathrm{min}^{-1} \mathrm{~A}^{-1}$ & 809 & 2.2 \\
\hline SOC range / \% & 90 & 53 \\
\hline Area $/ \mathrm{cm}^{2}$ & 2990 & 1400 \\
\hline Electrode Thickness / mm & 0.13 & 0.13 \\
\hline
\end{tabular}

OPTIMIZED PARAMETERS FOR 2M 2E NONAQEUOUS SYSTEM

interdigitated flow are much higher than the corresponding cost of $200 \$ \mathrm{kWh}^{-1}$ and 190 $\$ \mathrm{kWh}^{-1}$ for the all vanadium system.

Despite the poor performance at present cost for the non-aqueous system for both low and high E/P case, the high E/P case does show promise. The non-aqueous system may be able to use divalent active species, and use a higher concentration thus cutting costs on the expensive electrolyte solvent. This drops the cost for $\mathrm{E} / \mathrm{P}=0.1$-h to 4500 $\$ \mathrm{kWh}^{-1}$, similar to the all vanadium system. For $\mathrm{E} / \mathrm{P}=12-\mathrm{h}$, the system cost is $400 \$$ $\mathrm{kWh}^{-1}$ for conventional flow (twice of the all vanadium system cost) and $300 \$ \mathrm{kWh}^{-1}$ for interdigitated flow (50\% higher than all vanadium system cost). The flow pattern did not affect the low E/P ratio significantly, since pump costs start dominating at very high flow rates, thus removing the advantage of interdigitated flow pattern. However, for a high E/P ratio, higher flow rates are enabled with interdigitated flow design, due to lower pressure drop for this flow design. Hence, a higher SOC range is enabled, which limits energy costs.

Using $2 \mathrm{M}$ concentration for the electroactive species and 2 electrons per molecule, the cost is drastically decreased for the 12 -h case, reducing it by $72 \%$ for the present 


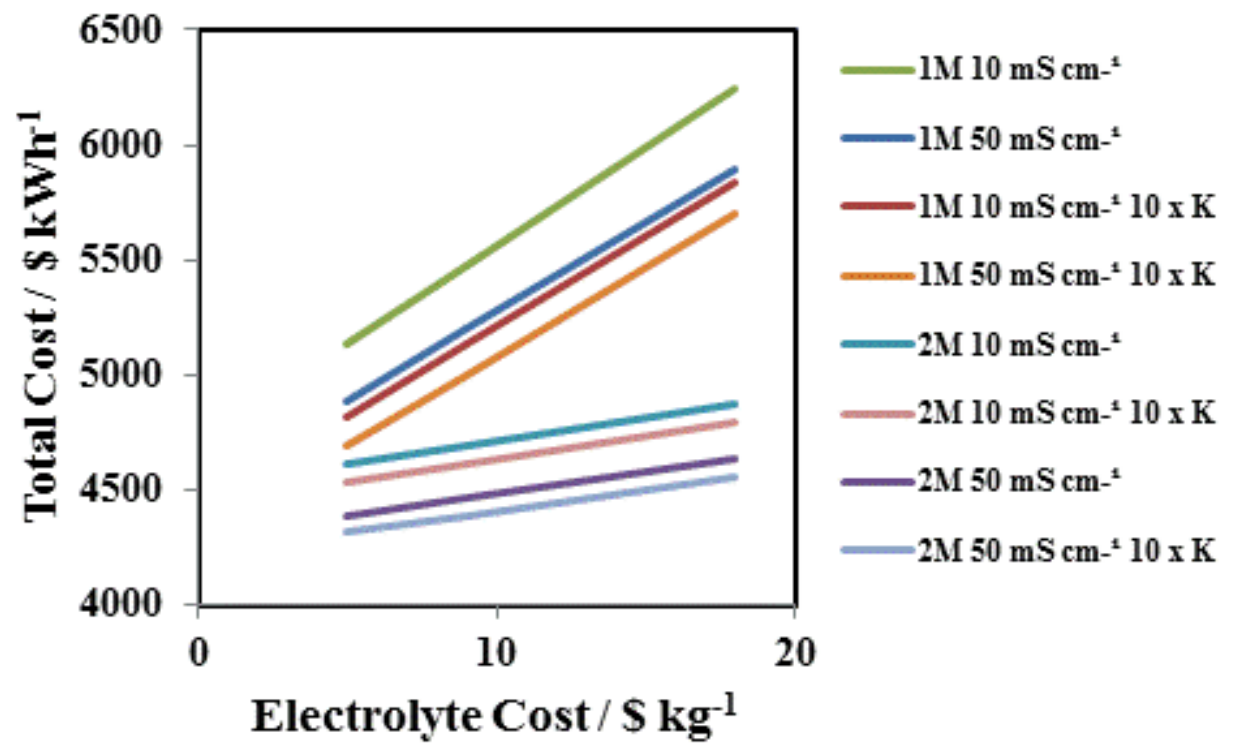

Figure 5.3. Parametric analysis for present case for $0.1-\mathrm{h}$

\begin{tabular}{|c|c|c|}
\hline Scenario & 12-h Opt thickness / mm & 0.1-h Opt thickness / mm \\
\hline $1 \mathrm{M} 32 \mathrm{mS} \mathrm{cm}^{-1}$ & 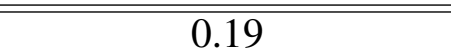 & $\bar{~} 0.17$ \\
\hline $2 \mathrm{M} 32 \mathrm{mS} \mathrm{cm}^{-1}$ & 0.13 & 0.13 \\
\hline $1 \mathrm{M} 50 \mathrm{mS} \mathrm{cm}^{-1}$ & 0.25 & 0.20 \\
\hline $1 \mathrm{M} 10 \mathrm{mS} \mathrm{cm}^{-1}$ & 0.08 & 0.06 \\
\hline $2 \mathrm{M} 50 \mathrm{mS} \mathrm{cm}^{-1}$ & 0.20 & 0.14 \\
\hline $2 \mathrm{M} 10 \mathrm{mS} \mathrm{cm}^{-1}$ & 0.06 & 0.04 \\
\hline $1 \mathrm{M} 32 \mathrm{mS} \mathrm{cm} \mathrm{cm}^{-1} 10 \times$ kinetics & 0.10 & 0.09 \\
\hline $1 \mathrm{M} 10 \mathrm{mS} \mathrm{cm}^{-1} 10 \times$ kinetics & 0.06 & 0.04 \\
\hline $2 \mathrm{M} 50 \mathrm{mS} \mathrm{cm}-110 \times$ kinetics & 0.07 & 0.06 \\
\hline
\end{tabular}

OPTIMIZED ELECTRODE THICKNESSES FOR PARAMETRIC ANALYSIS

case. This case effectively cuts energy costs by $75 \%$ even before taking into account the improved performance from the increased concentration due to lower contribution of SOC enabling a higher operating SOC range. 


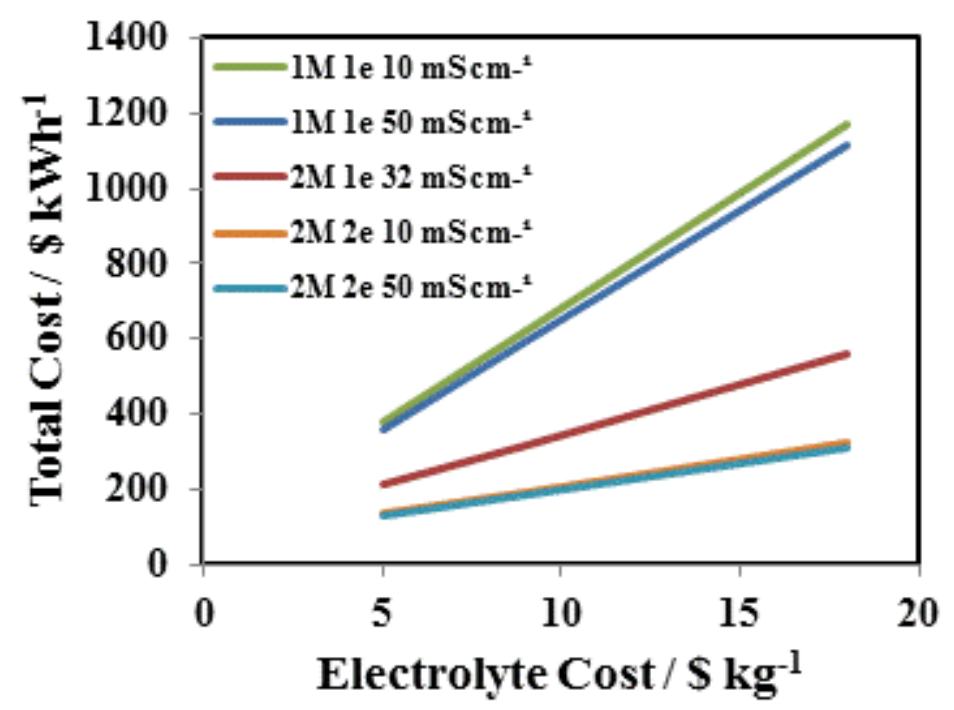

Figure 5.4. Parametric analysis for present case for 12-h

\section{Parametric Analysis}

A parametric study was also performed to analyze where efforts towards cost reduction could best be made. This was done for E/P ratios of $0.1-\mathrm{h}$ and 12-h. Fig 5.3 shows the results at various electrolyte costs, and changes in the parameters of conductivity and kinetics. To provide a fair comparison for each parameter change, the electrode thickness was also changed to minimize cost.

Fig 6a shows that electrolyte cost is still a major driver of cost, although this effect is reduced for higher concentrations. However it is not as important as the 12-h case (Fig. 5.4) as expected. Conductivity however does have a higher effect, due to the higher power and current density used. Increasing the conductivity 5-fold to $50 \mathrm{mS} \mathrm{cm}^{-1}$ decreases the cost by $\sim 8 \%$ for $1 \mathrm{M}$ concentration, with a reduction of $4 \%$ for $2 \mathrm{M}$ electrolyte. Increasing the concentration to $2 \mathrm{M}$ reduces cost by $10 \%$ and $20 \%$ at the low and high end of the electrolyte cost range. 
Increasing the kinetics of the reaction has a greater effect at low conductivity, with a cost reduction of 7 to $10 \%$ across the electrolyte cost range, with cost reduction increasing with electrolyte cost. At electrolyte conductivity of $50 \mathrm{mS} \mathrm{cm}^{-1}$, the cost reduction is only $3 \%$ across the electrolyte cost range.

For $2 \mathrm{M}$ electrolyte, increasing the conductivity lowers cost by $5 \%$, half of the decrease at $1 \mathrm{M}$. Increasing kinetic rate constant for baseline conductivity lowers the cost by $1.6 \%$, while the reduction is $1.4 \%$ for $50 \mathrm{mS} \mathrm{cm}^{-1}$. This figure highlights the interaction among kinetic rate constant, conductivity and concentration that determines system cost at various electrolyte cost. Clearly, in the order of priority, increasing the concentration (and electrons/mole) of the electroactive species is of paramount importance. Increasing the conductivity is significant mainly for low concentration, while improving kinetics is mainly significant for low concentration and conductivity. For all scenarios, lowering electrolyte cost is very important, with this becoming critical at low concentration of the electroactive species.

For the 12-h case, changing the conductivity does not change the cost very much compared to other improvements. For the $1 \mathrm{M}$ case $50 \mathrm{mS} \mathrm{cm}^{-1}$ corresponds to costs only $4 \%$ lower than $10 \mathrm{mS} \mathrm{cm}^{-1}$, with only a $3 \%$ drop in the $2 \mathrm{M}$ case. This is mainly due to the lower current being optimal at the high E/P.

Changing the kinetics also does not have much of an effect, with an increase in kinetics of $10 \mathrm{x}$ reducing the cost by $6 \%$ for $1 \mathrm{M}$ case and less than $1 \%$ for $2 \mathrm{M}$ case. This is partially due to adjusting the electrode thickness that minimizes the cost as the kinetic rate constant changes. When the kinetic parameter is extremely high, this lends itself to a reaction zone 
thickness that is very small. However, at very small electrode thickness, the pressure drop related losses are quite high, thus limiting how much the reaction zone can be shrunk. With our current design of interdigitated flow pattern, there was not much benefit in increasing the charge transfer rate. However, this is more of an engineering issues, and it is anticipated that with better options for limiting pressure drop, the higher charge transfer rate can be instrumental in cost reduction even for the 12-h operation. Clearly, for the high $\mathrm{E} / \mathrm{P}$ case, increasing electroactive species concentration and electrons per mole are the most important levers for cost reduction. The electrolyte cost, understandably, is more important for the high energy case. 


\section{CONCLUSION}

The models developed for estimating redox flow battery performance have shown their utility when validated vs stack data. The ability to model performance based on the physical parameters of conductivity and kinetic rate constant without possessing any stack data makes it straight forward to investigate and model novel battery chemistries. The model performs very well at estimating the physical performance of the V-V system. When turned into a computationally cheap algorithm, it can be used within an optimization routine to return the proper cost of the battery system while also providing guidance on how to operate the system.

The model can also provide guidance on which parameters are most important for reducing system cost, by way of parametric analysis. This was demonstrated by applying it to a novel non-aqueous system to investigate the effects of concentration, conductivity, and kinetics on system cost, allowing the strengths of this chemistry to be evaluated and areas promising for improvement to be identified.

\section{A. Future Work}

As the current distribution model has many benefits over the effective thickness model as discussed previously, future work in flow battery modeling should incorporate this, and move away from the effective thickness model.

Other future work would be to validate this model vs more experimental data, both to confirm it and to understand where improvements can be made. This experimental data could be for novel battery chemistries, or even changes such as improved kinetics or conductivity, changed electrode thickness, or electrolyte concentration. 
One major physical phenomenon ignored in the current distribution are effects resulting from the concentration gradient. As the reaction distribution is not treated as constant, varying utilization across the electrode thickness would result in a change in concentration with distance from the membrane. This would result in a changing Nernst potential across the electrode thickness that is not taken into account in the characteristic equations of the current distribution model.

Taking this effect into account would act as a result in a potential difference across the electrode proportional to the concentration gradient, which would be greater with less uniform current distribution, and lower with more uniform current distribution. Therefore this effect would make the current distribution more uniform across the electrode to offset this potential difference, resulting in a lower SOC drop at low SOC values. Due to the more uniform current distribution, the ionic current has a higher average distance to travel, so it would possibly increase ohmic losses in the middle of the SOC range. This could explain why the current distribution model under-predicts at low SOC and over-predicts at mid-SOC. Future work could incorporate this effect as a perturbation term to the current distribution solution. As this is a change in potential across the electrode thickness based on the local volumetric current distribution, this effect could be accounted for by increasing the charge transfer resistance for purposes of calculating the distribution. This would produce the desired effect of making the volumetric current density distribution more uniform. The adjusted characteristic equation would likely change from Eq 4.6 to the following: 


$$
\frac{d \eta}{d x}-\frac{d E_{n e r n s t}}{d x}=J \rho
$$

$$
\frac{d E_{n e r n s t}}{d x}=-\frac{R T}{F} \frac{d i}{d x} C_{T}\left(\frac{1}{S O C_{r x n}}+\frac{1}{1-S O C_{r x n}}\right)
$$

$$
\frac{d i}{d x}\left(\frac{d \eta}{d i}+\alpha\right)=J \rho
$$

Another phenomenon ignored in this model is how the SOC changes across the electrode height (in the direction of electrolyte flow). In this model the SOC at the cell outlet is used for modeling. However as the electrolyte is utilized across the cell height, another concentration gradient would ensue, with this concentration gradient being a function of current density, flow rate, cell dimensions, and diffusion constant. This could also be accounted for with a perturbation term to the current distribution solution.

Finally, the calculated current distribution and performance should be compared to the output of a finite element analysis model, using a commercially available software such as COMSOL. This could be used to test predictions about current distribution across the height of the cell and the thickness. This FEA validation could provide guidance on which phenomena are most important to account for. 


\section{REFERENCES}

[1] D. Aurbach and I. Weissman. Non Aqueous Electrochemistry. Marcel Dekker, Inc., 1999.

[2] E.V. Carino and F.R. Brushett. Preliminary investigation of the redox kinetics of 2,5-di-tert-butyl-1,4-bis(2methoxyethoxy)benzene (dbbb) using cyclic voltammetry data. V.V. Viswanathan, Editor, 2014.

[3] Alasdair Crawford. Pnnl cost-performance tool (doe-oe sponsored) for estimation of capital costs for redox flow batteries. https://github.com/PNNL-OE-Redox-Flow-Battery-Cost-Tool/ PNNL-OE-Redox-Flow-Battery-Cost-Tool, 2015.

[4] Alasdair Crawford, Vilayanur Viswanathan, David Stephenson, Wei Wang, Edwin Thomsen, David Reed, Bin Li, Patrick Balducci, Michael Kintner-Meyer, and Vincent Sprenkle. Comparative analysis for various redox flow batteries chemistries using a cost performance model. Journal of Power Sources, 293:388-399, 2015.

[5] Robert M Darling, Kevin G Gallagher, Jeffrey A Kowalski, Seungbum Ha, and Fikile R Brushett. Pathways to low-cost electrochemical energy storage: a comparison of aqueous and nonaqueous flow batteries. Energy \& Environmental Science, 7(11):3459-3477, 2014.

[6] Rachel Gelman. 2011 renewable energy data book. Technical report, National Renewable Energy Laboratory, 2013.

[7] Kevin L Gering. Prediction of electrolyte viscosity for aqueous and non-aqueous systems: Results from a molecular model based on ion solvation and a chemical physics framework. Electrochimica Acta, 51(15):31253138, 2006.

[8] Soowhan Kim, Edwin Thomsen, Guanguang Xia, Zimin Nie, Jie Bao, Kurtis Recknagle, Wei Wang, Vilayanur Viswanathan, Qingtao Luo, Xiaoliang Wei, et al. $1 \mathrm{kw} / 1 \mathrm{kwh}$ advanced vanadium redox flow battery utilizing mixed acid electrolytes. Journal of Power Sources, 237:300-309, 2013.

[9] David Stephenson, Soowhan Kim, Feng Chen, Edwin Thomsen, Vilayanur Viswanathan, Wei Wang, and Vincent Sprenkle. Electrochemical model of the fe/v redox flow battery. Journal of The Electrochemical Society, 159(12):A1993-A2000, 2012.

[10] Vilayanur Viswanathan, Alasdair Crawford, David Stephenson, Soowhan Kim, Wei Wang, Bin Li, Greg Coffey, Ed Thomsen, Gordon Graff, Patrick Balducci, et al. Cost and performance model for redox flow batteries. Journal of Power Sources, 247:1040-1051, 2014.

[11] Zhenguo Yang, Jianlu Zhang, Michael CW Kintner-Meyer, Xiaochuan Lu, Daiwon Choi, John P Lemmon, and Jun Liu. Electrochemical energy storage for green grid. Chemical reviews, 111(5):3577-3613, 2011. 
[12] Dongjiang You, Huamin Zhang, and Jian Chen. A simple model for the vanadium redox battery. Electrochimica Acta, 54(27):6827-6836, 2009. 